\title{
Catalogue of the Benjamin Balansa moss collections from Paraguay in Herbarium Genavense (G)
}

\author{
Michelle J. Price
}

\begin{abstract}
PRICE, M. J. (2012). Catalogue of the Benjamin Balansa moss collections from Paraguay in Herbarium Genavense (G). Candollea 67: 153-179. In English, English and French abstracts.

One of the most significant bryophyte collections from Paraguay is that of the Frenchman Benedict (Benjamin) Balansa. His collections were distributed to, amongst other institutions, B, BM, G, H-BR, L, NY and PC. In Geneva, Balansa collections can be found in the historical herbaria of Boissier, Reuter-Barbey, Barbey-Boissier and the non G-DC De Candolle collection which were all incorporated into the municipal herbarium $(G)$ of the Conservatoire et Jardin botaniques de la Ville de Genève. The French bryologist Emile Bescherelle worked on the majority of the Balansa moss collections from Paraguay, describing 73 new species from amongst this material. With the purchase of Bescherelle's herbarium by the BM, Balansa's original Paraguay specimens came to reside there. A total of 390 specimens are present in $\mathrm{G}$ for 158 Balansa collection numbers, including 22 specimens for 15 previously unpublished Balansa numbers. The 158 collection numbers and 390 specimens in G cover 88 specific and infraspecific names that represent 67 currently accepted taxa and eight nomina nuda. Of the 390 specimens, 160 are types including 3 lectotypes, 28 isolectotypes, 19 isotypes and 110 syntypes. For each taxonomic entry, the place of publication, the collection number, current name, and for the types the type status and typification, if found, are provided. A complete list of the
\end{abstract}

\begin{abstract}
Résumé
PRICE, M. J. (2012). Catalogue des collections de mousses de Benjamin Balansa du Paraguay dans l'Herbarium Genavense (G). Candollea 67 : 153179. En anglais, résumés en anglais et français.

L'une des collections de bryophytes du Paraguay les plus importantes est celle constituée par le français Benedict (Benjamin) Balansa. Ses collections ont été distribuées entre autres à $\mathrm{B}, \mathrm{BM}, \mathrm{G}, \mathrm{H}-\mathrm{BR}, \mathrm{L}, \mathrm{NY}$ et PC. A Genève, les collections de Balansa se trouvent dans les herbiers historiques Boissier, Reuter-Barbey, Barbey-Boissier ainsi que dans les collections De Candolle (non G-DC), toutes ces collections faisant partie de l'herbier général $(\mathrm{G})$ des Conservatoire et Jardin botaniques de la Ville de Genève. Le bryologiste français Emile Bescherelle a travaillé sur la plupart des collections de mousse de Balansa du Paraguay, décrivant 73 nouvelles espèces. A la suite de l'achat de l'herbier de Bescherelle par BM, les spécimens originaux de Balansa récoltés au Paraguay se trouvent maintenant conservés à cet endroit. Un total de 390 spécimens sont conservés à $\mathrm{G}$ représentant 158 numéros de la collection de Balansa, inclus 22 spécimens correspondant à 15 numéros de Balansa jamais publiés jusqu'ici. Les 158 numéros et 390 spécimens de $\mathrm{G}$ comprennent 88 noms spécifiques et infraspécifiques, représentant 67 taxa actuellement acceptés et 8 nomina nuda. De ces 390 spécimens, 160 sont des types parmi lesquels se trouvent 3 lectotypes, 28 isolectotypes, 19 isotypes et 110 syntypes. Pour chaque taxon, le lieu de
\end{abstract}

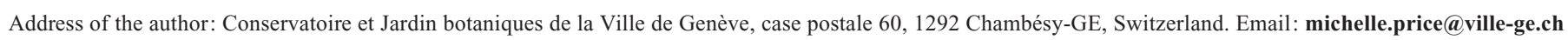


published Balansa collection numbers for the Paraguay mosses, including their current name, the name under which they were published and the place of publication, is also given herein.

\section{Key-words}

Paraguay - Mosses - Types - Catalogue - Benjamin Balansa South America. publication, le numéro de collecteur, le nom actuel et pour les types leur statut et typification (lorsque celle-ci a pu être trouvée), sont indiqués. Une liste complète des numéros de collection de Balansa pour les mousses du Paraguay comprenant le nom actuel, le nom sous lequel ils ont été publiés et le lieu de publication est également proposée. 


\section{Introduction}

A recent updated checklist of the mosses of Paraguay reported 240 species, including 8 infra-specific taxa, from 94 genera and 38 families (O’SHEA \& Price, 2008). One of the most significant bryophyte collections from Paraguay is that made by the Frenchman Benedict (Benjamin) Balansa during the late 1800 's. Considered to be a "scientific voyager", he spent over ten years living, working and collecting plants in Paraguay. He was based in the capital Asunción, at the invitation of the government at that time (FOURIER, 2005), but travelled and collected in the departments of Paraguarí, Guairá, and Caaguazú. Balansa collected plants from all groups during his two stays in Paraguay: the first between 1873 and 1877, and the second between 1878 and 1884 . His collections were distributed, amongst others, to B, BM, G, $\mathrm{H}-\mathrm{BR}, \mathrm{L}, \mathrm{NY}$ and PC.

The majority of the Balansa collections of Paraguayan mosses were worked on by the French bryologist Emile Bescherelle (1828-1903) who was based in Paris (BESCHERELLE, 1877, 1885, 1891a, 1891b, 1891c). BESCHERELLE (1885) consists of a list of the mosses distributed by Balansa in 1884, some names were of new species which were validated in his later series of publications "Selectio Novorum Muscorum" (BESCHERELLE 1891a, 1891b, 1891c). Bescherelle described 73 new names based on the Balansa Paraguayan collections, 20 of which are apparent endemics to Paraguay (Table 1).

Appendix 1 contains a complete list of the published Balansa Paraguay collection number series for mosses, including reference information, the name under which the collection number was published and the name under which each specimen is currently known. The presence/absence of material in $\mathrm{G}$ for each collection number is indicated alongside abridged information on the locality, taken from the first publication in which the number appeared. The place of the first publication of each collection number is also given. Of the 195 published collection numbers and 5 collections distributed without a number $\mathrm{G}$ holds 390 collections for 158 numbers.

A list of previously unpublished Balansa collection numbers that were found in $G$ is included at the end of the catalogue and in Appendix 1. This newly determined/verified material, accounts for 22 specimens for 15 previously unpublished Balansa collection numbers.

\section{The life of Benjamin Balansa (1825-1891)}

Benjamin Balansa, born Benedict Balansa, was the fourth son of Joseph and Jeanne (born Caussidou) Balansa. He was born on $28^{\text {th }}$ of March, 1825 in the commune of Narbonne (department Aude, region Languedoc-Roussillon), France. Evidence points to the fact that his mother was travelling to Toulouse on the Canal du Midi at the time of his birth as there are no records indicating that the family ever resided in
Narbonne (AstRE, 1947). According to the biography of Benjamin Balansa by Astre (1947) he studied in Sorèze (at the foot of Montagne-Noire in the department of Tarn) at 'Collège de Sorèze', and developed an interest in plants during his time there. When the family later moved to Paris he worked for two years as an editor at the 'Moniteur Universel'. In 1847, at the age of 22, he married Marie-Pauline Fabre and a year later began the activities that would occupy the next 44 years of his life, namely travelling, collecting plants and engaging in business ventures in foreign countries (for example, in Paraguay, Sicily, Turkey and Vietnam). He can be described as a "scientific voyager" as he had a particular interest in the exploration and exploitation of natural resources as well as in plant collecting (ASTRE, 1947). Many of his voyages were either conducted in the company of Edmond Bossier or where undertaken on his behalf. Balansa died in Hanoi, Vietnam, on the $22^{\text {nd }}$ November 1891 at the age of 66.

Voyages of B. Balansa (from Astre, 1947):

1847-1848 First voyage to Algeria,

1850-1853 Second voyage to Algeria,

1854-1855 First voyage to the Orient (Turkey and Sicily),

1856 Second voyage to the Orient ("Capadoce"),

1857-1865 Third voyage to the Orient (Turkey and Sicily),

1866 Voyage to Lazistan (Turkey),

1867 Voyage to Morocco (Mogador, Atlas Mountains, Marrakech),

1868-1872 Voyage to New Caledonia and the Loyalty Islands,

1873-1877 First voyage to Paraguay,

1878-1884 Second voyage to Paraguay,

1885-1889 First voyage to Tonkin (North Vietnam), with a trip to Java,

1890-1891 Second voyage to Tonkin (died in Hanoi).

\section{Catalogue of the Balansa moss collections from Paraguay in G}

In $\mathrm{G}$, the collections of Balansa from Paraguay are easily recognisable based on their distinctive labels. The collection series are entitled "B. Balansa - Pl. du Paraguay - 1874-1877" or "B. Balansa - Pl. du Paraguay - 1878-1884" and the labels themselves, written in French, generally have information on the locality and substrate for each specimen, the date (at least the year) the collection was made and a collection number. For many of the specimens in $\mathrm{G}$ a second number, the year of herbarium accession, has been added to the specimen label.

The original name under which the Balansa Paraguay collection numbers were published are listed alphabetically, with information on the place of publication and, for type specimens, 
Table 1. - The 73 taxa, including 4 infra-specific names and 14 nomina nuda, described by Emile Bescherelle from material collected in Paraguay by Benjamin Balansa. The 20 taxa that are currently listed as endemic to Paraguay are indicated in bold. The year of publication is included after the author.

Acrocryphaea paraguensis Besch. - $1891 \equiv$ Schoenobryum paraguense

(Besch.) Manuel

Anacalypta paraguensis Besch. - $1877 \equiv$ Uleastrum paraguensis (Besch.)

W. R. Buck

Aulacopilum paraguense Besch. - 1885 (nom. nud.)

Brachymenium spirale Besch. - 1891

Bruchia aurea Besch. - 1877

Bryum paraguense Besch. - 1877

Conomitrium polycarpum Besch. - 1891

Cryphaea guarapensis Besch. - 1885 (nom. nud.)

Cylindrothecium argyreum Besch. - 1891

Ectropothecium glaucinum Besch. - 1877 三 Vesicularia glaucina (Besch.) Broth.

Entosthodon balansae Besch. - 1877

Erpodium exsertum Besch. - 1891 = Erpodium biseriatum (Austin) Austin

Erpodium lanceolatum Besch. - 1885 (nom. nud.)

Erpodium paraguense Besch. -1877 = Erpodium beccarii Müll. Hal.

Fabronia balansaeana Besch. -1885 = Fabronia ciliaris var. polycarpa

(Hook.) W. R. Buck

Fabronia guarapensis Besch. - 1885 (nom. nud.)

Fabronia guarapiensis Besch. -1891 = Fabronia ciliaris var. polycarpa

(Hook.) W. R. Buck

Fabronia julacea Besch. -1877 = Fabronia ciliaris var. polycarpa (Hook.)

W. R. Buck

Fissidens balansaeanus Besch. -1877 = Fissidens zollingeri Mont.

Fissidens balansaeanus var. limosus Besch. $-1877=$ Fissidens zollingeri

Mont.

Fissidens brevipes Besch. - 1891 = Fissidens elegans Brid.

Fissidens glaucifrons Besch. -1891 = Fissidens elegans Brid.

Fissidens guarapiensis Besch. - 1891 = Fissidens zollingeri Mont.

Fissidens subcrispus Besch. $-1877=$ Fissidens crispus Mont.

Holomitrium paraguense Besch. - 1891

Homalia paraguensis Besch. - 1891 (nom. nud.)

Hookeria luteoviridis Besch. - 1891 = Thamniopsis langsdorffii (Hook.)

W. R. Buck

Hookeria subdepressa Besch. -1891 = Callicostella subdepressa (Besch.)

Broth.

Hymenostomum balansaeanum Besch. - 1877

Isopterygium guarapense Besch. - 1891 = Isopterygium tenerum (Sw.) Mitt. Isopterygium subtenerum Besch. -1891 = Isopterygium tenerum (Sw.) Mitt.

Lasia occulta Besch. -1891 = Forsstroemia occulta (Besch.) Paris Lasia paraguensis Besch. $-1877=$ Forsstroemia coronata (Mont.) Paris Lasia subcoronata Besch. - 1877 = Forsstroemia subcoronata (Besch.) Paris Lasia subcoronata var. patens Besch. - 1877 (nom. nud.)

Leptohymenium squarrosum var. griseum Besch. - $1877=$ Lorentziella paraguensis Besch. - 1877

Microdus paraguensis Besch. - 1891 = Leptotrichella paraguensis (Besch.)

Ochyra
Microthamnium eurystomum Besch. - 1877 = Mittenothamnium eurystomum (Besch.) Cardot

Microthamnium humile Besch. $-1877=$ Mittenothamnium humile (Besch.) Cardot

Neckera villae-ricae Besch. - 1877

Orthotrichum paraguense Besch. - 1891 = Sehnemobryum paraguense

(Besch.) Lewinsky-Haapasaari \& Hedenas

Papillaria guarapiensis Besch. - 1891

Papillaria subnigrescens Besch. - 1885 (nom. nud.)

Philonotis balansaeana Besch. - $1877=$ Philonotis curvata (Hampe) A. Jaeger

Philonotis balanseana Besch. - 1885 (nom. nud.)

Physcomitrium luteolum Besch. - 1877

Physcomitrium paraguense Besch. - 1877

Plagiothecium villae-ricae Besch. - 1877 = Isopterygium tenerifolium Mitt.

Pogonatum pallens Besch. - 1885 (nom. nud.)

Pseudoleskea paraguensis Besch. - 1877 = Haplocladium microphyllum (Hedw.) Broth.

Pterogoniopsis fabronia Besch. - 1885 = Sematophyllum fabronia (Besch.)

\section{W. R. Buck}

Ptychomitrium balansae Besch. -1877 = Ptychomitrium muelleri (Mitt.) A. Jaeger

Ptychomitrium mandoni Besch. - 1885 (nom. nud.)

Ptychomitrium subdentatum Besch. - 1877

Ptychomitrium vaginatum Besch. - 1877

Rhaphidostegium chlorothecium Besch. - 1877 ミSematophyllum chlorothecium (Besch.) Broth.

Rhaphidostegium fallax Besch. - 1877 = Sematophyllum subpinnatum (Brid.) E. Britton

Rhaphidostegium fusco-viride Besch. - 1891 = Sematophyllum subpinnatum (Brid.) E. Britton

Rhaphidostegium globosum Besch. - 1891 = Sematophyllum subpinnatum

(Brid.) E. Britton

Rhaphidostegium percircinale Besch. - 1891 (nom. nud.)

Rhynchostegium assumptionis Besch. - 1877

Rhynchostegium fissidentellum Besch. - 1877

Schlotheimia paraguensis Besch. - 1877

Schlotheimia paraguensis f. pruinosula Besch. - 1877 (nom. nud.)

Sphagnum flaccidum Besch. - 1877

Sphagnum paraguense Besch. - 1885 (nom. nud.)

Stereophyllum enerve Besch. - 1885 (nom. nud.)

Stereophyllum guarapense Besch. -1877 = Stereophyllum radiculosum (Hook.) Mitt.

Stereophyllum paraguense Besch. -1877 = Stereophyllum radiculosum (Hook.) Mitt.

Syrrhopodon paraguensis Besch. - 1891 = Syrrhopodon ligulatus Mont.

Thuidium paraguense Besch. - 1891

Trematodon palettifolius Müll. Hal. ex Besch. - 1877 
the type citation given. Publication details are given for specimens related to names originating from Paraguay material whereas publication information is not given where the name represents a straight determination of the material. For each record the basionym and/or published name is given alongside its modern name (in bold).

Information on the specimen labels is included for the type and non-type specimens. Label information for the first specimen entry of a series of specimens with the same collection number is given in full and if subsequent labels are identical then only the $\mathrm{G}$ barcode, type designation (if applicable) and herbarium of origin for each specimen is listed. If label information differs between labels with the same collection number then each variant is noted. When an herbarium accession year is present on the label this is indicated in square brackets.

Where a name has been typified then the published type designation and type location(s) are indicated, along with information on the G material. The terms lectotype, isolectotype, holotype and isotype are used when types have been specified and the term syntype is used herein wherever specific type designations were not found. For type ("Type specimens") and non-type specimens ("Specimens") the G barcode is given as an identifier. For non-type specimens the publication in which the collection number/s first appeared is given. Information on published nomina nuda is included and the specimens associated with these names are listed as "original material".

In G, Balansa collections can be found in the historical herbaria of Boissier, Reuter-Barbey, Barbey-Boissier, Duby, the non G-DC De Candolle collection as well as in the general collection. For any particular Balansa collection number present in $\mathrm{G}$ between 1 and 5 duplicates are found. Based on observations of the $\mathrm{G}$ collection, some of the Balansa duplicates appear to have been distributed without determinations as they have been annotated at a later date using the series of publications by BESCHERELLE (1877, 1885, 1891a, 1891b, 1891c).

The herbarium of origin of the specimen is noted using the following:

$\mathrm{G}=$ general herbarium of $\mathrm{G}$ (no specific source given on the label);

Herb. Barb.-Boiss. = Barbey-Boissier herbarium;

Herb. Boiss. $=$ Boissier herbarium;

Herb. DC. = De Candolle herbarium (non G-DC);

Herb. Duby = Duby herbarium;

Herb. Reuter-Barb. = Reuter-Barbey herbarium.

In this work all author names are standardized according to BRUMMIT \& POWELL (1992) and journal citations are according to the accepted standards of bryological literature (CROSBY, 1999). Accepted names are according to CROSBY \& al. (1999) and the Missouri Botanical Garden database Tropicos. Herbarium codes are according to Index Herbariorum.
Acrocryphaea paraguensis Besch. in J. Bot. (Morot) 5: 342. 1891.

= Schoenobryum paraguense (Besch.) Manuel in Bryologist 80: 523. 1977.

Type citation. - "Caraguazu, sur les arbres, janvier, Balansa 3628 ".

Type specimens. - Syntypes: Caaguazu, sur tronc des arbres, janvier 1882, Balansa 3628 (G [1885], G00040482; Herb. Boiss., G00040481 ; Herb. Boiss., G00040483 ; Herb. DC. [1884], G00040484).

Type designation. - Type designation not indicated in MANUEL (1977).

Amblystegium haplocladium Müll. Hal. in Hedwigia 36: 130. 1897.

Type citation. - "Paraguay, in sylvis prope Guarape truncos arborum habitans: B. Balansa 1878. Coll. No. 3679; L'assomption, in terra humosa, 27. Julio 1897: Balansa Coll. No. 3679".

Type specimens. - Syntypes: Forêt de Guarapi, sur le tronc des arbres 1878, Balansa 3679 (G [1885], G00048141; Herb. Boiss., G00042360; Herb. Boiss., G00048142; Herb. Boiss., G00048143; Herb. DC. [1884], G00048140). Type designation. - None found.

Comments. - Bescherelle (1885: 19) published this collection number with the name Microthamnium humile Besch.

Anacalypta paraguensis Besch. in Mém. Soc. Sci. Nat. Cherbourg $21: 261.1877$.

=Uleastrum paraguense (Besch.) W. R. Buck in Candollea 40: 203. 1985.

Type citation. - "Peragu [Pirayu], sur le tronc du Cocos yatai, Balansa 1220".

Type specimens. - Syntypes: Pirayu, sur le tronc du Cocos yataï, mai 1877, Balansa 1220 (G [1878], G00048151; Herb. Boiss., G00048152).

Type designation. - Type designation not indicated in BuCK (1985).

Aptychus circinicaulis Müll. Hal. in Hedwigia 36: 125. 1897.

= Sematophyllum circinicaule (Müll. Hal.) Broth. in Nat. Pflanzenfam. (ed. 2) 11:433. 1925.

Type citation. - "Paraguay, 'bords du Mbay', in truncis arborum, Julio 1881: B. Balansa in Coll. No. 3692". 
Type specimens. - Syntypes : Bords du Mbay, sur le tronc des arbres, juillet 1881, Balansa 3692 (G [1885], G00040506; Herb. Boiss., G00040496; Herb. Boiss., G00040497; Herb. DC. [1884], G00040505) [published in BESCHERELLE, 1885: 19].

Type designation. - None found.

Aulacopilum balansae Müll. Hal. in Flora 70: 447. 1887.

= Erpodium glaucum (Wilson) I. G. Stone, fide STONE (1997: 487).

Type citation. - "Guarapi, in truncis Aurantiorum, Junio 1881, Balansa 3643”.

Type specimens. - Syntypes: Guarapi, sur le tronc des orangers, juin 1881, Balansa 3643 (G [1885], G00042261; Herb. Boiss., G00042262 ; Herb. Boiss., G00042263; Herb. DC. [1884], G00042264).

Type designation. - None found.

Comments. - Number 3643 was also used as the original specimen by BESCHERELLE $(1885: 18)$ for the nomen nudum “Aulacopilum paraguense".

Barbula pallidoviridis Müll. Hal. in Hedwigia 36: 103. 1897.

= Tortella humilis (Hedw.) Jenn., fide CRUM \& STEERE (1957: 471).

Type citation. - "Paraguay 'bords du Mobay' in truncis arborum, Julio 1881, B. Balansa Coll. 3652 et $3652 a$ ".

Type specimens. - Syntypes : Bords du Mbay, sur le tronc des arbres, juillet 1881, Balansa 3652 (G [1885], G00042221; Herb. Boiss., G00042222 ; Herb. DC. [1884], G00042220).

Type designation. - None found.

Specimens. - Guarapi, sur le tronc des arbres, juin 1879, Balansa 3652a (G [1885], G00042219; Herb. Boiss., G00042223; Herb. DC. [1884], G00042218) [published in BESCHERELLE, 1885: 17].

Comments. - Number $3652 a$, with the locality "Bords du Mobay", was used by MülLER (1897) as one of the two types of Barbula pallidoviridis Müll. Hal. The G specimens for collection number $3652 a$ have different locality information and a different collection date than those cited in the protologue under the same number. The G material under number $3652 a$ is thus not treated as potential type material herein.
Braunia incana Müll. Hal.

= Braunia cirrhifolia (Mitt.) A. Jaeger, fide VAN DER WIJK \& al. (1959: 232).

Specimen. - Roches éruptives, au NE du Cerro de San Tomas, mars 1881, Balansa 3683 (G, G00040401) [published in BESCHERELLE, $1885: 17]$.

Bruchia aurea Besch. in Mém. Soc. Sci. Nat. Cherbourg 21 : 259. 1877.

Type citation. - "Assomption, juillet 1876, Balansa 1261". Type specimens. - Isotypes: L'Assomption, juillet 1876, Balansa 1261 (Herb. Boiss. [non-original Balansa label], G00046209; Herb. DC. [1878], G00046206).

Type designation. - RUSHING (1986: 57): holotype (BM), isotypes (G, H-BR, NY, PC, S).

Bruchia ligulata Müll. Hal. in Flora 71: 11. 1888.

= Bruchia aurea Besch., fide VAN DER WIJK \& al. (1959: 242).

Type citation. - "Paraguari, in terra humida camporum incultorum, Junio 1882; Assumtion [sic], Junio 1879, Balansa 3708 et $3658^{\prime}$.

Type specimens. - Syntypes: L'Assomption, juin 1879, Balansa 3658 (G [1885], G00046207; Herb. Boiss., G00046210; Herb. DC. [1884], G00046208). Paraguari, sur la terre humide, dans les champs en friche, 1882, Balansa 3708 (Herb. Barb.-Boiss., G00116002; Herb. DC. [1884], G00128216).

Type designation. - Type designation not indicated in RUSHING (1986).

Comments. - The collection number 3658 was published under Bruchia aurea Besch. by BESCHERELLE (1885: 17) before being used by Müller to describe B. ligulata .

Bryum argenteum var. corrugatum (Hampe) Besch.

= Bryum argenteum Hedw., fide CRUM \& STEERE (1957: 488).

Specimen. - L'Assomption - sur la terre, dans les clairières des broussailles, 14 août 1874, Balansa 1259 (Herb. DC., G00042200) [published in Bescherelle, 1877: 263].

Bryum corrugatum Hampe

= Bryum argenteum Hedw., fide OcHI (1980: 110). Specimens. - Guiaviti, sur la terre autour des habitations, 13 juillet 1881, Balansa 3647 (G [1885], G00042201; Herb. Boiss., G00042197; Herb. Boiss., G00042198; Herb. DC. [1884], G00042199) [published in BESCHERELLE, 1885: 18]. 


\section{Bryum paraguense Besch.}

Specimens. - Guarapi, sur le talus des chemins, juillet 1881, Balansa 3651a (G [1885], G00042404; Herb. DC., G00042405). Guarapi, sur le talus des chemins, août 1881, Balansa 3651a (Herb. Boiss. [1885], G00042403) [published in BESCHERELLE, 1885: 18].

Campylopus amabilis (Müll. Hal.) Paris

= Campylopus pyriformis (Schultz) Brid., fide FRAHM (1982: 49).

Specimens. - Cordillère de Péribebui, dans les terrains dénudés, 1879, Balansa 3660 (G [1885], G00048097; Herb. Boiss., G00048096; Herb. DC. [1884], G00048095) [published in BESCHERELLE, 1885: 17].

Cryphaea guarapensis Besch. in Rev. Bryol. 12: 18. 1885 [nom. nud.].

Type citation. - "Guarapi, Balansa 3629a".

Original specimen. - Guarapi, sur le tronc des arbres, 30 juillet 1881, Balansa 3629a (Herb. Boiss., G00042390).

Cylindrothecium argyreum Besch. in J. Bot. (Morot) 5: 346 . 1891.

$\equiv$ Entodon argyreus (Besch.) Broth. in Bih. Kongl. Svenska Vetensk.-Akad. Handl. 26. Afd. 3(7): 49. 1900.

Type citation. - "Guarapi, sur les troncs des arbres 1878, Balansa 3678".

Type specimens. - Syntypes: Guarapi, sur le tronc des arbres, 1878, Balansa 3678 (G, G00042391; G [1885], G00042392 ; Herb. Boiss., G00042396; Herb. Boiss., G00042397; Herb. DC. [1884], G00042393).

Type designation. - None found.

Dimerodontium balansae Müll. Hal. in Mém. Soc. Sci. Nat. Cherbourg 21:267. 1877.

Type citation. - "Assomption, sur les troncs d'arbres, Balansa 1207; Villa-Rica, forêt à l'Est de la Cordillère". Type specimens. - Isolectotypes: L'Assomption, sur le tronc des arbres, juin 1874, Balansa 1207 (G [1878], G00042237; G [1878], G00042238).

Type designation. - CÂMARA \& MAGILl (2009: 305): lectotype (PC), isolectotypes (BM, G, NY).

Specimens. - Guarapi, Balansa 3702 (G, G00042239; G, G00042240; G, G00042241 ; G [1884], G00042242) [published in BESCHERELle, 1885: 18]. Guarapi, Balansa 3681 (G, G00042244) [published in Müller \& StEPHANI, 1887: 57].
Dusenia cuspidata Müll. Hal. in Hedwigia 36: 108. 1897.

$=$ Forsstroemia trichomitria (Hedw.) Lindb., fide STARK (1987: 191), see note in O'SheA \& Price (2008: 30).

Type citation. - "Paraguay, sine loco natali, Balansa Coll. No. 3662".

Type specimens. - Lectotype: Guarapi, sur le tronc des arbres, 10 août 1881, Balansa 3662 (G [1885], G000 42104). Isolectotypes: (Herb. Boiss., G00042103; Herb. DC. [1884], G00042102).

Type designation. - StARK (1987: 191): lectotype (G). Comments. - Number 3662 was named "Lasia subcoronata Besch." by BESCHERELLE (1885: 18) before being used by Müller (1897) as the type of Dusenia cuspidata.

Ectropothecium glaucinum Besch. in Mém. Soc. Sci. Nat. Cherbourg 21:271. 1877.

= Vesicularia glaucina (Besch.) Broth. in Engl. \& Prantl, Nat. Pflanzenfam. I(3): 1094. 1908.

Type citation. - "Caaguazu, Balansa 1198; Assomption, Balansa 1271".

Type specimens. - Syntypes: Caaguazu, dans les forêts, 24 mars 1876, Balansa 1198 (Herb. Boiss., G00042207; Herb. DC. [1878], G00042208).

Type designation. - None found.

Specimens. - Guarapi, sur les troncs des arbres, juin 1881, Balansa 3691 (Herb. Boiss., G00042204; Herb. DC. [1884], G00042203) [published in BESCHERELLE, 1885: 19].

Entodon denticulatum Müll. Hal. in Rev. Bryol. 14: 57.1887 [nom. nud.].

= Erythrodontium longisetum (Hook.) Paris, fide MAJestyk (2009: 808).

Type citation. - "s.1., Balansa 3678a".

Original specimen. - Town in Caaguazu, Paraguay, 1884, Balansa 3678a (Herb. Boiss. [non-original Balansa label], G00042394).

Entosthodon balansae Besch. in Mém. Soc. Sci. Nat. Cherbourg 21: 263. 1877.

Type citation. - "Assomption, sur la terre, dans les clairières, Balansa 1227; sur les talus, Balansa 1228; Villa-Rica, Balansa 1233; Guarapi, Balansa 1226".

Type specimens. - Syntypes: Guarapi, sur la terre humide, juin 1877, Balansa 1226 (Herb. Boiss., G00042519; Herb. DC. [1878], G00042520; Herb. Reuter-Barb., G00042535). L'Assomption, sur la terre, dans les clairières des broussailles, 14 août 1874, Balansa 1227 (Herb. Boiss., 
G00042521; Herb. DC. [1878], G00042522). L'Assomption, sur les talus des chemins, août 1876, Balansa 1228 (Herb. Boiss., G00042523 ; Herb. DC. [1878], G0004 2524).

Type designation. - None found.

Specimens. - L'Assomption, sur les talus des chemins ombragés, juillet 1881, Balansa 3649 (G [1885], G000 42527; Herb. Boiss., G00042525 ; Herb. Boiss., G0004 2528; Herb. DC. [1884], G00042526) [published in BESCHERELLE, 1885: 18].

\section{Ephemerum conicum Müll. Hal.}

Specimens. - Guarapi, sur la terre humide, 1878, Balansa 3621 (G, G00040404; G [1885], G00040405; Herb. DC. [1884], G00040406) [published in BESCHERELLE, 1885: 17].

Erpodium balansae Müll. Hal. in Flora 70: 449. 1887.

= Erpodium glaziovii Hampe, fide CRUM (1972: 216).

Type citation. - "Guarapi et Assumtion in truncis Aurantorium Junio 1881 et 1884 , Balansa 3645 et 3645 a. Cum Aulacopilo Balansae associatum".

Type specimens. - Syntypes: Guarapi, sur le tronc des orangers, juin 1881, Balansa 3645 (Herb. Boiss., G00042229; Herb. DC. [1884], G00042345; Herb. DC [1885], G00042346). L'Assomption, sur le tronc des orangers, juin 1881, Balansa 3645a (G [1885], G00042226; Herb. Boiss., G00042227 ; Herb. Boiss., G00042228; Herb. DC. [1884], G00042225).

Type designation. - None found.

Comments. - Number $3645 a$ was published as "Erpodium coronatum Hook. et Wils., Assomption" by BESCHERELLE (1885: 18) before being used later by Müller as the type of E. balansae.

Erpodium exsertum Besch. in J. Bot. (Morot) 5 : 254. 1891.

= Erpodium biseriatum (Austin) Austin, fide CRUM (1972: 209)

Type citation. - "Péribébuy, Balansa 3646".

Type specimens. - Syntypes: Cordillère de Péribébui, sur le tronc des arbres, juillet 1879, Balansa 3646 (Herb. Boiss., G00042273 ; Herb. Boiss., G00042274 ; Herb. DC. [1884], G00042275).

Type designation. - CRUM (1972: 210): type (PC [Crum!]), type designation not indicated.
Erpodium lanceolatum Besch. in Rev. Bryol. 12: 18. 1885 [nom. nud.].

= Erpodium beccarii Müll. Hal., fide CRUM (1972: 211).

Type citation. - "Guarapi, Balansa 3644”.

Original specimens. - Guarapi, sur le tronc des orangers, juin 1881, Balansa 3644 (G, G00042231 ; G, G00042234 ; G [1884], G00042232; G [1885], G00042233).

Comments. - CRUM (1972: 212) indicated that this collection number was found under different names in different herbaria.

Fabronia balansae Besch. in J. Bot. (Morot) 5: 345. 1891.

= Fabronia ciliaris var. polycarpa (Hook.) W. R. Buck, fide BuCK (1983: 251).

Type citation. - "Guarapi, associé au Dimerodontium balansae, 1881, Balansa 3656".

Type specimens. - Isolectotypes: Guarapi, 1881, Balansa 3656 (G, G00042333; G [1885], G00042334 ; Herb. DC. [1884], G00042335).

Type designation. - BucK (1983: 251): lectotype (BM), isolectotype $(\mathrm{G})$.

Fabronia guarapiensis Besch. in J. Bot. (Morot) 5: 345. 1891.

= Fabronia ciliaris var. polycarpa (Hook.) W. R. Buck, fide BucK (1983: 251).

Type citation. - "Guarapi, sur le tronc des arbres, 14 juillet 1881, Balansa 3681, associé au Dimerodontium Balansae et à divers Frullania et autres Jungermannes".

Type specimens. - Isolectotypes: Guarapi, sur le tronc des arbres, 14 juillet 1881, Balansa 3681 (G, G00042243 ; Herb. Boiss., G00042336; Herb. Boiss., G00042337). Type designation. - BucK (1983: 251): lectotype (BM), isolectotypes ( $\mathrm{G}[2])$.

Fabronia julacea Besch. in Mém. Soc. Sci. Nat. Cherbourg $21: 265.1877$.

= Fabronia ciliaris var. polycarpa (Hook.) W. R. Buck, fide BuCK (1983: 251).

Type citation. - "Assomption, sur les troncs des arbres, Balansa 1256".

Type specimens. - Syntypes: L'Assomption, sur le tronc des arbres, avril 1877, Balansa 1256 (G, G00042530; $\mathrm{G}$ [1878], G00046104).

Type designation. - BucK (1983: 251): types (BM, NY), holotype location not indicated. 
Fissidens balansaeanus Besch. in Mém. Soc. Sci. Nat. Cherbourg $21: 260.1877$.

= Fissidens zollingeri Mont., fide PURSELL (1994: 266). Type citation. - "Assomption, parois des puits, août 1874, Balansa 1241".

Type specimens. - Isolectotypes: L'Assomption, sur les parois d'un puits, 8 août 1874, Balansa 1241 (Herb. Boiss., G00042254; Herb. DC. [1878], G00042253).

Type designation. - PURSELL (1994: 266): lectotype (BM). PuRSELl (2007: 225): holotype (BM).

Fissidens balansaeanus var. limosus Besch. in Mém. Soc. Sci. Nat. Cherbourg 21: 261. 1877.

= Fissidens zollingeri Mont., fide Pursell (2007: 225).

Type citation. - "Yaguarou [Yaguaron], sur la terre, Balansa $1245^{\prime \prime}$

Type specimens. - Isolectotypes: Yaguaron, 1877, Balansa 1245 (Herb. Boiss., G00042255; Herb. DC. [1878], G00114035).

Type designation. - Pursell (2007: 225): lectotype (S) "Yaguarou, 1877, Balansa s.n.".

Fissidens brevipes Besch. in J. Bot. (Morot) 5: 252. 1891. Type citation. - "Paraguay, ad cortices, Balansa 3698". Type specimen. - Isolectotype: Guarapi, dans les forêts, Balansa 3698 (Herb. Boiss., G00042276).

Type designation. - PURSELL (1994: 256): lectotype (NY).

Fissidens glaucifrons Besch. in J. Bot. (Morot) 5: 252. 1891.

= Fissidens elegans Brid., fide Pursell (1994: 258).

Type citation. - "Assomption, talus, Balansa 3697".

Type specimens. - Lectotype: L'Assomption sur le talus des chemins, juillet 1879, Balansa 3697 (Herb. Boiss., G00042250). Isolectotypes: (Herb. Boiss., G00042251; Herb. DC. [1884], G00042252).

Type designation. - PURSELl (1994: 258): lectotype (G), isolectotypes (G, NY).

Fissidens guarapiensis Besch. in J. Bot. (Morot) 5: 252. 1891.

= Fissidens zollingeri Mont., fide PuRSELL (1994: 266). Type citation. - "Guarapi, sur la terre, Balansa 3699". Type specimens. - Isolectotypes: Guarapi, sur la terre, avril 1881, Balansa 3699 (G [1885], G00042249; Herb. Boiss., G00042245; Herb. Boiss., G00042246; Herb. Boiss. [nonoriginal Balansa label], G00042247; Herb. DC. [1884], G00042248).
Type designation. - PURSELL (1994: 266): lectotype (BM). PURSELl (2007: 226): holotype (BM), isotype (NY).

Comments. - Number 3699 was used by Müller (MÜLLER \& Stephani, 1887: 57) for the nomen nudum "Fissidens stenocarpus".

Fissidens subcrispus Besch. in Mém. Soc. Sci. Nat. Cherbourg 21:260. 1877.

= Fissidens crispus Mont., fide PURSell (1994: 256).

Type citation. - "Assomption, sur les pelouses, juillet 1876, Balansa 1202".

Type specimens. - Isolectotypes: L'Assomption, sur les pelouses, juillet 1876, Balansa 1202 (G [1878], G0012 1791; Herb. DC., G00040407).

Type designation. - PURSELL (1994: 256): lectotype (HBR). PuRsell (2007: 78): holotype (BM), isotypes (H-BR, NY, S).

Specimens. - Bords du Mbaii, sur le tronc des arbres, juillet 1881, Balansa 3637 (G [1885], G00048101 ; Herb. Boiss., G00048098; Herb. Boiss., G00048099; Herb. DC. [1884], G00048100) [published in BeSCHERELLE, 1885: 17).

Funaria calvescens Schwägr.

= Funaria hygrometrica var. calvescens (Schwägr.) Mont., fide BowERs (1970: 17).

Specimens. - Paraguari, sur la terre humide, juillet 1881, Balansa 3653 (G, G00040410; G [1884], G00040409; G [1885], G00040411; Herb. Boiss., G00040408). Guarapi, dans les défrichés nouvellement incendiés, juin 1879, Balansa 3653a (G [1885], G00040412 ; Herb. Boiss., G00040413; Herb. DC. [1884], G00040414). L'Assomption, sur la terre humide, juillet 1879, Balansa $3653 b$ (Herb. Boiss., G00040415) [published in BesCherelle, 1885: 18).

\section{Helicodontium capillare (Hedw.) A. Jaeger}

Specimen. - L'Assomption, 1876, Balansa 1208 (Herb. DC. [1878], G00042367) [published in Bescherelle, 1877: 268].

\section{Helicodontium pervirens (Müll. Hal.) Paris}

Specimens. - Guarapi, sur le tronc des arbres, 1881, Balansa 3687 (G [1885], G00042362 ; Herb. Boiss., G000 42364; Herb. Boiss., G00042365; Herb. DC. [1884], G000 42363) [published in BESCHERELLE, 1885:18]. 
Hookeria luteoviridis Besch. in J. Bot. (Morot) 5: 344. 1891.

= Thamniopsis langsdorffii (Hook.) W. R. Buck, fide BuCK (1987: 218).

Type citation. - "Cerro Léon, près de Pirayu, sur les troncs d'arbres, 23 juillet 1881, Balansa 3638".

Type specimens. - Isotypes: Cerro Léon, près de Pirayu, sur l'écorce des arbres, 23 juillet 1881, Balansa 3638 (G [1885], G00042182; Herb. Boiss., G00042184; Herb. DC. [1884], G00042183).

Type designation. - Buck (1987: 218): holotype (BM).

Hookeria subdepressa Besch. in J. Bot. (Morot) 5: 345. 1891.

$\equiv$ Callicostella subdepressa (Besch.) Broth. in Engl. \& Prantl, Nat. Pflanzenfam. I(3): 937. 1907.

Type citation. - "Guarapi, 1881, Balansa 3689".

Type specimens. - Syntypes: Paraguay: Guarapi, dans les forêts, 1881, Balansa 3689 (G[1885], G00042270; Herb. Barb.-Boiss., G00042271 ; Herb. Boiss., G00042272; Herb. DC. [1884], G00042269).

Type designation. - None found.

Comments. - Number 3689 was used by Müller (MÜLLER \& StePhani, 1887: 57) as original material for the nomen nudum "Hookeria paraguensis".

\section{Hymenostomum balansaeanum Besch.}

Specimens. - Bopicua entre Paraguari et Yaguaron, sur le talus des chemins, juillet 1881, Balansa 3700 (G [1885], G00042348; Herb. Boiss., G00042352; Herb. DC. [1884], G00042350) [published in BesCherelle, 1885: 17].

Hypnum circinicaule Müll. Hal. in Rev. Bryol. 14: 57.1887 [nom. nud.].

= Sematophyllum circinicaule (Müll. Hal.) Broth. in Engl., Nat. Pflanzenfam. ed. 2, 11: 433. 1925.

Type citation. - "Sin. loc., Balansa 3692".

Original specimen. - 3692. Hypnum circinicaulis C. Müller n. sp. Peribebuy (Paraguay) Balansa (Herb. Barb.-Boiss. [non-original Balansa label], G00048085).

Comments. - A specimen numbered 3692 from the locality "Bords du Mbaï" was named "Rhaphidostegium circinale (Hpe)" by BESCHERELLE (1885: 19) and later served as the type of Aptychus circinicaulis Müll. Hal. (= Sematophyllum circinicaule (Müll. Hal.) Broth.), see under that name. Since the above specimen does not have an original Balansa label, and is assumed to be possibly erroneous, this specimen is not included in Appendix 1.
Hypnum nanum Müll. Hal. in Rev. Bryol. 14: 57.1887 [nom. nud.].

Type citation. - "Sin. loc., Balansa 3695".

Original specimen. - Guarapi, sur le tronc des arbres, juillet 1881, Balansa 3695 (Herb. Boiss., G00040495).

Hypopterygium argentinicum Lorentz ex Müll. Hal.

= Hypopterygium tamarisci $(\mathrm{Sw}$.$) Brid. ex Müll. Hal., fide$ KRUIJER (2002: 202).

Specimens. - Guarapi, dans les forêts, sur le tronc des arbres, août 1879, Balansa 3628a (G [1885], G00048104; Herb. Barb.-Boiss., G00048105; Herb. Boiss., G0004 8103; Herb. DC. [1884], G00048102). Cordillère de Mbatobi, près de Paraguari, sur les roches humides, juin 1881, Balansa 3629 (G [1885], G00048106; Herb. Barb.-Boiss., G00048107; Herb. Boiss., G00048108; Herb. DC. [1884], G00048109). Guarapi, sur le tronc abattu des arbres, dans les forêts, 8 avril 1879, Balansa 3630 (Herb. Barb.-Boiss., G00048110 ; Herb. Boiss., G00048111) [published in BESCHERELLE, 1885: 18].

Isopterygium guarapense Besch. in J. Bot. (Morot) 5: 349 . 1891.

= Isopterygium tenerum $(\mathrm{Sw}$.$) Mitt., fide IRELAND (1991:$ 270).

Type citation. - "Guarapi, sur les écorces d'arbres, 1878, Balansa 3619".

Type specimens. - Isotypes: Guarapi, sur les écorces des arbres, 1878, Balansa 3619 (Herb. Boiss., G00042532; Herb. DC. [1884], G00042531).

Type designation. - IRELAND (1991: 270): holotype (BM), isotype $(\mathrm{H})$.

Isopterygium subtenerum Besch. in J. Bot. (Morot) 5: 348 . 1891.

= Isopterygium tenerum $(\mathrm{Sw}$.$) Mitt., fide IRELAND (1991:$ 270).

Type citation. - "Cordillère de Péribébui, juillet 1879, Balansa 3690".

Type specimens. - Isotypes: Cordillère de Péribébui, juillet 1879, Balansa 3690 (G [1885], G00040418; Herb. Boiss., G00040416; Herb. DC. [1884], G00040417). Type designation. - IRELAND (1991: 270): holotype (BM). 
Lasia occulta Besch. in J. Bot. (Morot) 5: 342. 1891.

= Forsstroemia trichomitria $($ Hedw.) Lindb., fide STARK (1987: 191).

Type citation. - "Guarapi, sur les troncs d'arbres, Balansa 3663".

Type specimens. - Isolectotypes: Guarapi, sur le tronc des arbres, 10 août 1881, Balansa 3663 (G [1884], G0004 2101; Herb. Boiss., G00042100; Herb. DC. [1884], G000 42099).

Type designation. - STARK (1987: 191): lectotype (PC).

Lasia paraguensis Besch. in Mém. Soc. Sci. Nat. Cherbourg 21:266. 1877.

= Forsstroemia coronata (Mont.) Paris, fide STARK (1987: 177).

Type citation. - "Yaguaron, dans les forêts, sur les troncs d'arbres, Balansa 1252".

Type specimen. - Lectotype: Forêts de Yaguarone, juin 1877, Balansa 1252 (Herb. DC. [1878], G00042093).

Type designation. - StARK (1987: 177): lectotype (G). Specimens. - Cerro Léon, près de Pirayu, sur l'écorce des arbres, 23 juillet 1881, Balansa 3667 (Herb. Boiss., G00042097; Herb. DC. [1884], G00042098). Bords du Mbai, sur les branches des arbres, juillet 1881, Balansa 3668 (G [1885], G00042368; Herb. Boiss., G00042369; Herb. DC. [1884], G00042370). Cordillère de Péribebui, sur les écorces des arbres, 1879, Balansa 3669 (G [1885], G00042096; Herb. Boiss., G00042094; Herb. DC. [1884], G00042095) [published in BesCHERELLE, $1885: 18$ ].

\section{Leptohymenium squarrosum Hampe}

$\equiv$ Erythrodontium squarrosum (Hampe) Paris, Index Bryol. (ed. 2) 2: 159. 1904.

Specimens. - Cordillère de Mbatobi, près de Paraguari, sur le tronc des arbres, 26 juin 1881, Balansa 3666 (G [1885], G00042382 ; Herb. Boiss., G00042383 ; Herb. Boiss., G00042384; Herb. DC. [1884], G00042381). Bords du Mbay, sur le tronc des arbres, juillet 1881, Balansa 3676 (G [1885], G00042388; Herb. Boiss., G00042385; Herb. Boiss., G00042386; Herb. DC., G00042387). Forêts Paraguari, Balansa 3676 (Herb. Boiss. [non-original Balansa label], G00042395) [published in BeSCHERELLE, 1885: 19].
Lorentziella paraguensis Besch. in Mém. Soc. Sci. Nat. Cherbourg 21:259. 1877.

= Lorentziella imbricata (Mitt.) Broth., fide LAWTON (1953: 287).

Type citation. - "Assomption, sur les pelouses, juillet 1876, Balansa 1258".

Type specimens. - Syntypes: L'Assomption, sur les pelouses, juillet 1876, Balansa 1258 (Herb. Boiss., G00046141; Herb. DC. [1878], G00040419).

Type designation. - LAWTON (1953: 281): types (NY, PC), holotype location not indicated.

Specimens. - L'Assomption, dans les clairières des broussailles, juin 1881, Balansa 3623 (G [1885], G00046142; Herb. Boiss., G00046143; Herb. DC. [1884], G00046140) [published in BESCHERELLE, 1885: 17].

Macromitrium phyllorhizans Müll. Hal. ex Besch. in Mém. Soc. Sci. Nat. Cherbourg 21: 262. 1877.

= Macrocoma orthotrichoides (Raddi) Wijk \& Margad., fide ViтT (1980: 413).

Type citation. - "Pirayu, sur les troncs d'arbres, Balansa 1237; Paraguari dans les forêts, Balansa 1240".

Type specimen. - Isolectotype: Perayu, dans les forêts, sur le tronc des arbres, mai 1877, Balansa 1237 (Herb. DC. [1878], G00048069).

Type designation. - VitT (1980: 413): lectotype (NY). Specimens. - Cordillère de Péribébui, juillet 1879, Balansa 3665 (G [1885], G00048124; Herb. Boiss., G00048117; Herb. DC. [1884], G00048121). Guarapi, sur les tronc des arbres, 1881, Balansa 3670 (G [1885], G00048125; Herb. Boiss., G000 48118; Herb. Boiss., G00048119; Herb. DC. [1884], G0004 8120). Cordillère de Péribébui, juillet 1879, Balansa 3671 (Herb. DC., G00048123) [published in BESCHERELLE, 1885: 18].

Meiothecium fabronia Besch. in J. Bot. (Morot) 5: 343. 1891.

$\equiv$ Sematophyllum fabronia (Besch.) W. R. Buck in Contr. Univ. Michigan Herb. 15: 139. 1982.

Type citation. - "Cordillère de Péribébui, juillet 1879, Balansa 3693, associé au Rhaphidostegium (Aptychus) percircinale C. Muell.".

Type specimens. - Syntypes: Cordillère de Péribébui, juillet 1879, Balansa 3693 (G [1885], G00042265; Herb. Boiss., G00042266; Herb. Boiss., G00048077; Herb. DC. [1884], G00048076).

Type designation. - Type designation not indicated in BuCK (1982).

Comments. - Number 3693 was used by Müller (MÜLLER \& STEPHANI, 1887: 57) as original material for the earlier nomen nudum "Hypnum percircinale". 
Microdus paraguensis Besch. in J. Bot. (Morot) 5: 145. 1891.

$\equiv$ Leptotrichella paraguensis (Besch.) Ochyra in Fragm. Florist. Geobot. 42: 563. 1997.

Type citation. - "Paraguay: Péribébui, Balansa 3661".

Type specimens. - Syntypes: Cordillère de Peribébui, dans les terrains dénudés, juillet 1879, Balansa 3661 (G [1885], G00040428; Herb. Boiss., G00040425 ; Herb. Boiss., G00040427; Herb. DC., G00042267).

Type designation. - Type designation not indicated in OCHYRA (1997).

Comments. - Number 3661 was also used as the type for Conomitrium polycarpum Besch. (= Fissidens hornschuchii Mont.) with locality information "Paraguay" by BESCHERELLE (1891b: 252).

Specimen. - Yaguaron, sur les berges humides des ruisseaux, juillet 1879, Balansa 3659 (Herb. Boiss., G00040424).

Microthamnium eurystomum Besch. in Mém. Soc. Sci. Nat. Cherbourg 21:269. 1877.

= Mittenothamnium eurystomum (Besch.) Cardot in Rev. Bryol. 40: 21. 1913.

Type citation. - "Villa-Rica, Balansa 1199, 1209, 1278". Type specimens. - Syntypes: Villa-Rica, dans les forêts sur la terre humide, septembre 1874, Balansa 1199 (Herb. DC., G00048082). Villa-Rica, dans les forêts, septembre 1874, Balansa 1209 (Herb. Boiss., G00048080; Herb. DC. [1878], G00048081).

Type designation. - None found.

Specimens. - Cerro-Leon, près de Diraya, sur l'écorce des arbres, 23 juillet 1881, Balansa 3639 (G [1885], G0004 8129; Herb. Boiss., G00048132; Herb. DC., G00048127). Guarapi, dans les bois, 1879, Balansa 3640a (Herb. Boiss., G00048126; Herb. Boiss., G00048130). Guarapi, dans les bois, mars 1880, Balansa 364la (Herb. Boiss., G00048131; Herb. DC. [1884], G00048128) [published in BesCHERElle, 1885: 19].

Neckera balansae Müll. Hal. in Hedwigia 36: 107. 1897.

= Neckeropsis disticha (Hedw.) Kindb., fide VAN DER WIJK \& al. (1964: 435).

Type citation. - "Paraguay, Guarapi, in truncis arboretum; 1884, Balansa 3627".

Type specimens. - Syntypes: Guarapi, sur le tronc des arbres, Balansa 3627 (G [1885], G00040434; Herb. Boiss., G00040432; Herb. DC. [1884], G00040433).

Type designation. - None found.
Neckeropsis undulata (Hedw.) Reichardt

Specimens. - Forêts situées à l'est de la Cordilliére de VillaRica, 30 septembre 1877, Balansa 1255 (Herb. DC. [1878], G00040445) [published in Bescherelle, 1877: 265]. Cordillère de Villa-Rica, dans les forêts vierges, tronc des arbres, octobre 1877, Balansa 1255 (Herb. Boiss., G0004 0444). Guarapi, sur l'écorce des arbres, août 1881, Balansa 3625 (G [1885], G00040436; Herb. Boiss., G00040435; Herb. Boiss., G00040438; Herb. DC. [1884], G00040437). Cordillère de Mbatobi, près de Paraguari, sur le tronc des arbres, 26 juin 1881, Balansa 3626 (G [1885], G00040440; Herb. Boiss., G00040439; Herb. Boiss., G00040442; Herb. DC. [1884], G00040441) [published in BESCHERELLE, 1885: 18)].

Octoblepharum albidum Hedw.

Specimens. - Guarapi, dans les forêts, sur le tronc des arbres, mars 1880, Balansa 3654 (G [1885], G00040448; Herb. DC. [1884], G00040447; Herb. Duby, G00040446) [published in BesCHERELLE, 1885: 17]. Ecorce des arbres. Cordillère de Mbatobi, 24 mai 1883, Balansa 4256 (Herb. Barb.-Boiss., G00040450; Herb. DC. [1885], G00040451; Herb. DC. [1885], G00040452 ; Herb. Duby, G00040449) [published in MüLler \& StePHANI, 1887: 57].

Papillaria subnigrescens Besch. in Rev. Bryol. 12: 17. 1885 [nom. nud.].

Type citation. - "Guarapi, Balansa 3664, Paraguari, Balansa 3666a".

Original specimens. - Guarapi, sur le tronc des arbres, 1881, Balansa 3664 (Herb. Boiss., G00040430; Herb. Boiss., G00040431). Cordillère de Mbatobi, près de Paraguari sur le tronc des arbres, 26 juin 1881, Balansa $3666 a$ (G [1885], G00040422 ; Herb. Boiss., G00040420; Herb. DC. [1884], G00040421).

Phascum recurvirostrum Müll. Hal. in Flora 71: 5. 1888.

$\equiv$ Tetrapterum recurvirostre (Müll. Hal.) Broth. in Engl., Nat. Pflanzenfam. ed. 2, 10: 253. 1924.

Type citation. - "Yuguaron [Yaguaron], ad vias cavas, Julio 1879, Balansa 3657".

Type specimens. - Syntypes: Yaguaron, sur le talus des chemins, juillet 1879, Balansa 3657 (G [1885], G00042349; Herb. Boiss., G00042347; Herb. DC. [1884], G00042351).

Type designation. - None found.

Comments. - Number 3657 was published as Hymenostomum balanseanum Besch. in Bescherelle (1885: 17) before being used as the type of Phascum recurvirostrum by Müller. 
Philonotis balansaeana Besch. in Mém. Soc. Sci. Nat. Cherbourg 21:264. 1877.

= Philonotis curvata (Hampe) A. Jaeger, fide DISMIER (1910: 8).

Type citation. - "Villa-Rica, sur la terre, septembre 1874, Balansa 1195".

Type specimens. - Syntypes: Villa-Rica, sur la terre, septembre 1874, Balansa 1195 (Herb. DC. [1878], G00040 393). Forêts situées à l'est de la Cordillère de Villa-Rica, 30 septembre 1874, Balansa 1195 (Herb. Boiss., G00040394).

Type designation. - None found.

Philonotula oreadea Müll. Hal. in Hedwigia 36: 99. 1897.

= Philonotis uncinata (Schwägr.) Brid., fide GRIFFIN (1994: 560).

Type citation. - "Paraguay, Paraguari, ad declivitates viarum, Julio 1881: B. Balansa. Coll. No. 3655".

Type specimens. - Syntypes: Paraguari, sur le talus des chemins, juillet 1881, Balansa 3655 (G [1885], G0004 0395; Herb. Boiss., G00040397; Herb. Boiss., G0004 0398; Herb. DC. [1884], G00040396) [published in BESCHERELLE, 1885: 18].

Type designation. - None found.

Comments. - Number 3655 was published by BESCHERELLE (1885: 18) as Philonotis balansaeana Besch. before being used by Müller as the type of Philonotula oreadea.

Physcomitrium luteolum Besch. in Mém. Soc. Sci. Nat. Cherbourg $21: 263.1877$.

Type citation. - "Assomption, talus ombragés, Balansa $1230 "$ ".

Type specimens. - Syntypes: Talus ombragés des chemins, L'Assomption, 20 août 1876, Balansa 1230 (Herb. Boiss., G00046204). L'Assomption, sur les talus des chemins ombragés, 20 août 1876, Balansa 1230 (Herb. DC. [1878], G00046205).

Type designation. - None found.

Specimens. - Guarapi, sur la terre humide, 10 août 1881, Balansa 3648 (G, G00046212; G [1885], G00042195; Herb. Boiss., G00042193; Herb. DC. [1884], G00042194) [published in BESCHERELLE, 1885: 18].
Physcomitrium paraguense Besch. in Mém. Soc. Sci. Nat. Cherbourg 21:261. 1877.

Type citation. - "Assomption, bords des mares desséchées et sur les pelouses, Balansa 1229, 1231, 1232".

Type specimens. - Syntypes: L'Assomption, sur les pelouses, juillet 1876, Balansa 1229 (Herb. Boiss., G00042185; Herb. DC. [1878], G00042187). L'Assomption, bord des mares desséchées, 1876, Balansa 1231 (Herb. DC. [1878], G00042188; Herb. DC. [1878], G00042189).

Type designation. - None found.

Specimens. - L'Assomption, sur la terre humide, septembre 1878, Balansa 3650 (G [1885], G00042191). Cordillère de Péribébui, sur la terre humide, juillet 1879, Balansa $3650 b$ (Herb. Boiss., G00042186; Herb. Boiss., G00042192; Herb. DC. [1884], G00042190) [published in BesCHERELle, 1885: 18].

Plagiothecium villae-ricae Besch. in Mém. Soc. Sci. Nat. Cherbourg 21:271. 1877.

= Isopterygium tenerifolium Mitt., fide BUCK \& IRELAND (1989: 19).

Type citation. - "Villa-Rica, Balansa 1210".

Type specimens. - Isotypes: Villa-Rica, dans les forêts, septembre 1774, Balansa 1210 (Herb. Boiss., G00046238; Herb. DC. [1878], G00046235).

Type designation. - BucK \& IRELAND (1989: 19): holotype (BM).

Specimens. - Cordillère de Péribebui, dans les terrains humides ombragés, juillet 1879, Balansa 3636 (G, G00046236; Herb. Boiss., G00046237; Herb. DC. [1884], G00046239) [published in BESCHERELLE, 1885: 19].

Polytrichum perpusillum Müll. Hal. in Hedwigia 36: 90. 1897.

= Pogonatum pensilvanicum (Hedw.) P. Beauv., fide O'SHEA \& Price (2008: 36).

Type citation. - "Paraguay, Caraguaza, ad ripas rivulorum humidas et umbrosas, Febr. 1884, Balansa 3618".

Type specimens. - Isotypes: Caaguazu, sur les berges humides et ombragées des ruisseaux, février 1882, Balansa 3618 (G [1885], G00048075; Herb. Boiss., G00048068; Herb. DC. [1884], G00048074).

Type designation. - HYvÖNEN (1989: 18): isotype (H-BR), holotype location not indicated.

Comments. - Number 3618 , Pogonatum pallens Besch. in Rev. Bryol. 2: 18. 1885. nom. nud. was later used as a type by MüLLER (1897: 90) for Polytrichum perpusillum Müll. Hal. See discussion in O'SHEA \& PRICE (2008: 36). 
Pseudoleskea paraguensis Besch. in Mém. Soc. Sci. Nat. Cherbourg 21:267. 1877.

= Haplocladium microphyllum (Hedw.) Broth., fide REIMERS (1937: 228).

Type citation. - "Assomption, Balansa 1201, 1203, 1204, 1205, 1206, 1211; Villa-Rica, Balansa 1200".

Type specimens. - Syntypes: Villa-Rica, dans les forêts, septembre 1874, Balansa 1200 (Herb. DC. [1878], G00042359). L'Assomption, sur les talus des chemins ombragés, septembre 1875, Balansa 1201 (Herb. DC., G00042358). L'Assomption, 1876, Balansa 1203 (Herb. DC. [1878], G00042354). L'Assomption, août 1874, Balansa 1204 (Herb. DC. [1878], G00042353). L'Assomption, mai 1877, Balansa 1205 (Herb. DC. [1878], G00042356). L'Assomption, mai 1874, Balansa 1206 (G, G00042361; Herb. DC. [1878], G00042355). L'Assomption, 1876, Balansa 1211 (Herb. DC. [1878], G00042357).

Type designation. - None found.

Specimens. - L'Assomption sur la terre humide, 27 juillet 1879, Balansa 3679a (G [1884], G00046263; G [1885], G00046265; Herb. Boiss., G00046262 ; Herb. Boiss., G00046264) [published in BESCHERELLE, 1885: 18).

Comments. - Number $3679 a$ also appears in MÜLLER \& StePHANi (1887: 14) under the nomen nudum "Hypnum (Tamariscella) subnudum var.".

Ptychomitrium balansae Besch. in Mém. Soc. Sci. Nat. Cherbourg $21: 261.1877$.

= Ptychomitrium muelleri (Mitt.) A. Jaeger, fide CAO \& al. (2001: 522).

Type citation. - "Yaguaron, sur les toits, mars 1877, Balansa $1235^{\prime \prime}$.

Type specimens. - Syntypes: Yaguaron, sur les toits, mai 1877, Balansa 1235 (Herb. Boiss., G00040454; Herb. DC. [1878], G00040453).

Type designation. - CAO \& al. (2001: 522): isotype (BM), holotype location not indicated.

Ptychomitrium vaginatum Besch. in Mém. Soc. Sci. Nat. Cherbourg 21:262. 1877.

Type citation. - "Cerro Hu, dans les forêts, sur les rochers, Balansa 1234".

Type specimen. - Syntype: Rochers dans les forêts du Cerro-Hu, près de Paragarí, juin 1874, Balansa 1234 (Herb. DC. [1878], G00114192).

Type designation. - None found.
Specimens. - Yaguaron, sur les toits, 6 juillet 1881, Balansa 3672 (G [1885], G00048115; Herb. Boiss., G000 48112; Herb. DC. [1884], G00048116). Roches verticals courounant le Cerro Hu, près de Paraguari, 10 mai 1881, Balansa 3674 (G [1885], G00048122 ; Herb. Boiss., G00048113; Herb. DC. [1884], G00048114) [published in BESCHERELLE, 1885: 18].

\section{Racopilum tomentosum (Hedw.) Brid.}

Specimens. - L'Assomption, sur la terre humide, 12 septembre 1875, Balansa 1221 (G, G00040457; Herb. DC. [1878], G00048084). L'Assomption, 1876, Balansa 1221 (Herb. Reuter-Barb., G00040456). Forêts situées a l'est de la Cordillère de Villa-Rica, 30 septembre 1874, Balansa 1222 (G, G00040462; Herb. DC. [1878], G00048087). Forêts à l'Est de la Cordillère de Villa-Rica, 29 septembre 1874, Balansa 1224 (Herb. DC. [1878], G00048086) [published in BesChERELLE, 1877: 267]. Bojicua, entre Paraguari et Yaguaron, sur le talus des chemins, juillet 1881, Balansa 3677 (G, G00040459; G [1885], G00040458; Herb. Boiss., G00048090; Herb. DC. [1884], G00048088). VillaRica, sur la margelle d'un puits, 13 janvier 1881, Balansa 3685 (G, G00040461; G [1885], G00040460; Herb. Boiss., G00048091; Herb. DC. [1884], G00048089) [published in BesCHERELLE, 1885: 18].

\section{Rhaphidostegium aureolum (Hampe) A. Jaeger}

= Sematophyllum subpinnatum (Brid.) E. Britton, see O'Shea \& PRICE (2008: 36).

Specimens. - Guarapi, sur le tronc des arbres, juillet 1881, Balansa 3695 a (Herb. Boiss., G00040494; Herb. DC. [1884], G00040490) [published in BesCherelle, 1885: 19].

\section{Rhaphidostegium chlorothecium Besch.}

= Sematophyllum chlorothecium (Besch.) Broth. in Engl., Nat. Pflanzenfam. ed. 2, 11: 433. 1925.

Specimens. - Guarapi, sur le tronc des arbres, 29 juillet 1881, Balansa 3696 (Herb. Boiss., G00048093 ; Herb. Boiss., G00048094; Herb. DC. [1884], G00048092) [published in BESCHERELLE, 1885: 19].

Rhaphidostegium circinale (Hampe) Besch.

= Sematophyllum subpinnatum (Brid.) E. Britton, see O'SHEa \& PRICE (2008: 36).

Specimen. - L'Assomption, 1874, Balansa 1218 (Herb. DC. [1878], G00040493) [published in Bescherelle, 1877: 270]. 
Rhaphidostegium fallax Besch. in Mém. Soc. Sci. Nat. Cherbourg 21:270. 1877.

= Sematophyllum subpinnatum (Brid.) E. Britton, see O’ShEa \& PRICE (2008: 36).

Type citation. - "Assomption, sur les troncs d'arbres, Balansa 1213; Pirayu, sur le tronc du Cocos yatai, Balansa $1219 "$.

Type specimen. - Syntype: L'Assomption, sur le tronc des arbres, mai 1877, Balansa 1213 (Herb. DC. [1878], G00040488).

Type designation. - None found.

Specimens. - Cordillère de Peribébui, 1879, Balansa 3694 (Herb. Boiss., G00040498; Herb. Boiss., G00040504) [published in BESCHERELLE, 1885: 19].

Rhaphidostegium fuscoviride Besch. in J. Bot. (Morot) 5: 347 . 1891.

= Sematophyllum subpinnatum (Brid.) E. Britton, see O'SHEA \& PRICE (2008: 36).

Type citation. - "Cordillère de Péribébui, Balansa 3682".

Type specimens. - Syntypes: Cordillère de Peribébui, 1879, Balansa 3682 (G, G00040492; G, G00040500; Herb. Boiss., G00040501).

Type designation. - None found.

Specimen. - Cordillère de Peribébui, 1879, Balansa $3682 b$ (Herb. Boiss., G00040499) [published in BesCHERELle, 1885: 19].

Rhaphidostegium globosum Besch. in J. Bot. (Morot) 5: 347. 1891.

= Sematophyllum subpinnatum (Brid.) E. Britton, see O'Shea \& PRICE (2008: 36).

Type citation. - "Paraguay, sur les rochers des cascades de la Cordillère de Mbatobi près de Paraguari, Balansa 3633".

Type specimens. - Syntypes: Roches des cascades de la Cordillère de Mbatobi, près de Paraguari, mai 1881, Balansa 3633 (G [1885], G00040507; Herb. Boiss., G00040502; Herb. Boiss., G00040503; Herb. DC. [1884], G00040491). Type designation. - None found.

\section{Rhaphidostegium?}

= Sematophyllum subpinnatum (Brid.) E. Britton Specimens. - Cerro Leon, près de Pirayu, sur le tronc des arbres, 23 juillet 1881, Balansa 3635 (G, G00048072; G, G00048073; Herb. DC. [1884], G00048071; Herb. DC. [1885], G00048070) [published in BESCHERELLE, 1885: 19].
Rhynchostegium assumptionis Besch.

Specimens. - Cordillère du Cerro Péron, près de Pirayu, juillet 1880, Balansa 3632 (G, G00042560; G [1885], G00040468; Herb. Boiss., G00040467; Herb. Boiss., G00040469). Guarapi, dans les bois, 1879, Balansa 3642 (G, G00040471; G [1885], G00040473; Herb. Boiss., G0004 0472; Herb. DC. [1884], G00040470). Guarapi, sur le tronc des arbres, juin 1881, Balansa 3701 (G [1885], G00040477; Herb. Boiss., G00040475; Herb. DC., G00040474) [published in BESCHERELLE, 1885: 19].

Rhynchostegium fissidentellum Besch. in Mém. Soc. Sci. Nat. Cherbourg 21:270. 1877.

Type citation. - "Guarapi, sur la terre ferrugineuse, Balansa 1217".

Type specimen. - Syntype: Guarapi, 1877, Balansa 1217 (Herb. DC. [1878], G00040476).

Type designation. - None found.

Schlotheimia argentinica Lorentz \& Müll. Hal.

Specimens. - Guarapi, sur le tronc des arbres, 1881, Balansa 3675 (G, G00042214; G [1884], G00042215; Herb. Boiss., G00040478; Herb. Boiss. [1885], G00040479) [published in Bescherelle, 1885: 18].

Comments. - Number 3675 was named "Schlotheimia paraguensis Besch.” by Müller (MÜller \& StePhanI, 1887: 57).

Schlotheimia paraguensis Besch. in Mém. Soc. Sci. Nat. Cherbourg 21:262. 1877.

Type citation. - "Pirayu, sur les troncs d'arbres dans les forêts, mai 1877, Balansa 1236".

Type specimen. - Syntype: Pirayu dans les forêts, sur les troncs des arbres, mai 1877, Balansa 1236 (Herb. Boiss., G00042213).

Type designation. - None found.

Sphaerangium lorentzii (Müll. Hal.) Paris

= Astomum lorentzii (Müll. Hal.) Broth. in Engl. \& Prantl, Nat. Pflanzenfam. I(3): 397. 1902.

Specimen. - L'Assomption, dans les clairières des broussailles, juin 1881, Balansa 3624 (G [1885], G00042406) [published in BesCHERELLE, 1885: 17].

Comments. - Number 3624, with locality information of "Paraguay, summitate montis Cerro de Yuguaron, in terra planitiei, 17 junio 1879" was used as the type of Aucaulon nanum Müll. Hal. 
Sphagnum flaccidum Besch. in Mém. Soc. Sci. Nat. Cherbourg 21:272. 1877.

Type citation. - "Villa-Rica. Prairies marécageuses à l'Est de la Cordillère, septembre 1874, Balansa 1260".

Type specimens. - Isotypes: Prairies marécageuses à l'est de la Cordillère de Villa-Rica, 25 septembre 1874, Balansa 1260 (Herb. Boiss., G00042517; Herb. DC. [1878], G00042518). Type designation. - CRUM \& BucK (1988: 193): isotype (NY), holotype location not indicated.

Stereophyllum enerve Besch. in Rev. Bryol. 12: 19. 1885 [nom. nud.].

Type citation. - "Bords de l'Aroyo Piragu [Pirayu], Balansa 3634".

Original specimens. - Bords de l'Aroyo Pirayu, sur le tronc des arbres, 1 août 1880, Balansa 3634 (G [1885], G0004 2258; Herb. Boiss., G00042257; Herb. Boiss., G000422 59; Herb. DC. [1884], G00042260).

Stereophyllum guarapense Besch. in Mém. Soc. Sci. Nat. Cherbourg 21:269. 1877.

= Stereophyllum radiculosum (Hook.) Mitt., fide GrouT (1945: 61); IRELAND \& BuCK (1994: 4).

Type citation. - "Guarapi, Balansa 1216".

Type specimen. - Isotype: Guarapi, juin 1877, Balansa 1216 (Herb. DC. [1878], G00042202).

Type designation. - IRELAND \& BUCK (1994: 4): holotype (BM).

Specimens. - L'Assomption, Mai 1874, Balansa 1196 (G [1878], G00042584) [published in BESCHERELLE, 1877: 269]. Guarapi, sur le tronc des arbres, juin 1881, Balansa 3680 (G, G00048139; G [1885], G00048136; Herb. Barb.Boiss., G00048144; Herb. Boiss., G00048137; Herb. DC. [1884], G00048135). Guarapi, sur le tronc des orangers, 28 juillet 1881, Balansa 3682 a (G, G00048134; Herb. Boiss., G00048138) [published in BESCHERELLE, 1885: 19].

Comments. -Number 3680 was used by Müller (MÜLLER \& Stephani, 1887: 57) for the nomen nudum "Hypnum turgidicaule".
Stereophyllum homalioides Besch. in J. Bot. (Morot) 5: 252. 1891.

= Entodontopsis nitens (Mitt.) W. R. Buck \& R. R. Ireland, fide BucK \& Ireland (1985: 104).

Type citation. - "Cordillère de Peribébui, sur les écorces, juillet 1879, Balansa $3880 a$ ".

Type specimens. - Isotypes: Cordillère de Peribébui, juillet 1879, Balansa 3680a (Herb. Boiss., G00048078; Herb. Boiss., G00048079).

Type designation. - IRELAND \& BUCK (1994: 11): holotype (BM).

Comments. - Number $3680 a$ was published under the nomen nudum "Omalia paraguensis" by BESCHERELLE $(1885,18)$ with the same locality information.

Syrrhopodon paraguensis Besch. in J. Bot. (Morot) 5: 349 . 1891.

= Syrrhopodon ligulatus Mont., fide ReESE (1978: 203).

Type citation. - "Cerro de Mani, près de Paraguary, sur le tronc du Cocos australis, mars, 1888, Balansa 3673 R.R.R.”.

Type specimen. - Isotype: Cerro de Maui, près de Paraguari, sur le tronc du Cocos australis, mars 1880, Balansa 3673 (G, G00042407).

Type designation. - REESE (1978: 204): holotype (BM), isotypes (G, S-PA).

Comments. - Number 3673 was first published as "Syrrhopodon argentinicus C. Müll." by Bescherelle (1885: 17) before later being used as the type of S. paraguensis. Müller (MÜller \& StePhANI, 1887) cited the collection number 3673 for his nomen nudum "Hypnum subnudum", although it appears not to be based on the same material as that used to describe $S$. paraguensis as the locality information differs.

Thuidium paraguense Besch. in J. Bot. (Morot) 5: 346. 1891. Type citation. - "Guarapi, dans les forêts, sur les troncs pourris, juillet 1878, Balansa 3688, associé au Cylindrothecium argyreum Nob.".

Type specimen. - Syntype: Guarapi, dans les forêts, sur les troncs pourris, juillet 1878, Balansa 3688 (Herb. Boiss., G00042256).

Type designation. - None found. 
Trematodon palettifolius Besch. in Mém. Soc. Sci. Nat. Cherbourg 21:260. 1877.

Type citation. - "Villa-Rica, talus des chemins, septembre 1874, Balansa 1257. Proche du T. reflexus C. Müll.".

Type specimens. - Syntypes: Villa-Rica, Talus des chemins, 9 septembre 1874-1878, Balansa 1257 (Herb. DC. [1878], G00050525). s.1., s.d., Balansa 1257 (Herb. Boiss., G00042209).

Type designation. - None found.

Specimens. - L'Assomption, sur terre argileuse humide, juin 1879, Balansa 3631 (G, G00042212 ; Herb. DC., G00042210; Herb. DC. [1884], G00042211) [published in BESCHERELLE, 1885: 17].

\section{Previously unpublished Balansa collection numbers from Paraguay}

Names for 7 previously unpublished collection numbers and two collections without collection numbers (all unmarked) were taken for the herbarium label. New determinations are given for a further 8 collection numbers (marked with "§”).

\section{Bryum paraguense $\mathrm{Besch}$.}

Specimen. - Cordillère de Péribébui, juillet 1879, Balansa $3651 b$ (Herb. Boiss., G00042402).

Entodon argyreus (Besch.) Besch. §

Specimen. - Guarapi, dans les bois, 1881, Balansa 4265 (Herb. Barb.-Boiss., G00040402).

Entodontopsis nitens (Mitt.) W. R. Buck \& R. R. Ireland § Specimen. - Bords du Mbay, sur le tronc des arbres, juillet 1880, Balansa 4260 (Herb. Barb.-Boiss., G00040403).

Forsstroemia coronata (Mont.) Paris $\S$

Specimen. - Bords du Mbay, sur le tronc des arbres, juillet 1881, Balansa 4259 (G, G00042529).

\section{Haplocladium microphyllum (Hedw.) Broth. §}

Specimens. - Bords du Mbay, sur le tronc des arbres, juillet 1881, Balansa 4258 (Herb. Barb.-Boiss., G00042587). Yaguaron, sur le talus des chemins, juillet 1879, Balansa 4262 (Herb. Barb.-Boiss., G00042559).

\section{Leptotrichella paraguensis (Besch.) Ochyra}

Specimens. - Cordillère de Peribébui, dans les terrains dénudés, juillet 1879, Balansa 3661 (Herb. Barb.-Boiss., G00040429; Herb. Boiss., G00040426; Herb. DC. [1885], G00042572).
Pogonatum pensilvanicum (Hedw.) P. Beauv. §

Specimens. - Vallée de l'Y-acan guazu entre Paraguari et Valenzuela, sur les talus des chemins, 20 janvier 1884, Balansa 4332 (G, G00042224; Herb. DC. [1885], G00128743; Herb. DC. [1886], G00128740).

Ptychomitrium muelleri (Mitt.) A. Jaeger Specimen. - Paraguay, Balansa s.n. (G, G00040455).

Racopilum tomentosum (Hedw.) Brid.

Specimens. - Villa-Rica, dans les forêts, septembre 1874, Balansa 1194 (Herb. DC. [1878], G00040463). Guarapi, 1877, Balansa s.n. (Herb. DC. [1878], G00040464).

\section{Sphagnum magellanicum Brid.}

Specimens. - Pastoréo, près de Caaguazu, dans les prairies marécageuses, janvier 1882, Balansa 3616 (Herb. Boiss., G00046938; Herb. DC., G00042378).

Tortella pseudocaespitosa (Müll. Hal.) Broth.

Specimens. - Cordillère de Péribébui, juillet 1879, Balansa $3652 b$ (G [1885], G00042217). L'Assomption, sur les murs, septembre 1878, Balansa 3652c (Herb. Barb.-Boiss., G00042216).

Vesicularia glaucina (Besch.) Broth.

Specimens. - Guarapi, dans les bois, mars 1880, Balansa 3641 (G [1884], G00042578; Herb. Boiss., G00042205; Herb. Boiss., G00042206).

\section{Duplicated collection numbers within the Balansa Paraguay collection}

Within the Balansa Paraguay collection (see Appendix 1) 15 collection numbers are duplicated. For six of these duplicated collection numbers $(1199,3624,3661,3673,3679,3686)$ locality information differs and the taxonomic determinations of the material differ to the extent that it can be assumed that these numbers were assigned twice. For one of the numbers (3657) locality information is identical but given the taxonomic determinations of the specimens it can be assumed that this number was assigned twice. For the remainder of the numbers $(3619,3645 a, 3658,3675,3681,3687,3691,3699)$ the locality information is the same (or very similar) or missing for one of the duplicated numbers and the specimen determinations are close (mostly in same genus). This would indicate that the specimens with these collection numbers may well have been determined differently by different authors or that two close 
species were included within the original collection that was later split into duplicates. In some cases one of the duplicated numbers is a type for a particular name while the other represents a specimen of a different taxon.

\section{Conclusion}

In all 200 Balansa collection numbers for Paraguay have been located in the literature. G holds 158 of these collection numbers, as presented in Appendix 1. A total of 390 Balansa specimens are now known to be present in $\mathrm{G}$ for 88 names representing 67 currently accepted taxa and eight nomina nuda. Of the 390 Balansa specimens located to date in G 160 are types: 3 lectotypes, 28 isolectotypes, 19 isotypes and 110 syntypes. Seventeen original specimens for the 8 nomina nuda were also located. A total of 22 specimens for 15 previously unpublished Balansa collection numbers, representing 12 taxa, were enumerated herein.

As pointed out by BUCK (1985) the moss flora of Paraguay is poorly known, mainly owing to under-collecting within the country. This is echoed in the number of types that were encountered during this work for which no published designation could be found and in the high number of endemic moss taxa that are still listed for Paraguay. O'SHEA \& PRICE (2008) highlighted that very few collections are known from the departments of Canindeyú, Ñeembecú, Misiones and San Pedro. These four departments contain areas that are potentially interesting bryologically given their location, topography and/or humidity levels (see ZARDINI, 1993 for an overview of the habitat types in Paraguay).

The widely distributed moss collection of Benjamin Balansa from Paraguay, with its 200 published numbers, is one of the most important of the historical bryophyte collections from Paraguay and one that has made a significant contribution to the understanding of mosses in the country. This catalogue of the published moss collections of Balansa from Paraguay in $\mathrm{G}$ and the full listing of the Balansa collection numbers linked to their current names (Appendix 1) provides information that should facilitate the integration of specimens that were distributed without determinations into herbarium collections as well as the inclusion of these collections in future taxonomic work on South American mosses.

\section{Acknowledgements}

This project was supported by Conservatoire et Jardin botaniques de la Ville de Genève $(\mathrm{G})$. I thank Eva Maier (CJB) for her comments on an earlier version of this manuscript and for her invaluable contribution to bryology-based research at G. I especially thank Brian O'Shea for his permission to use and adapt his original list of Balansa Paraguay collection numbers for this work. I am grateful to Len Ellis for providing a review of this manuscript and Lorenzo Ramella for his collaboration on the manuscript. Lorenzo Ramella kindly provided the French translation of the abstract.

\section{References}

Astre, G. (1947). La vie de Benjamin Balansa, botaniste explorateur. Douladoure, Toulouse, France.

Bescherelle, E. (1877). Notes sur les Mousses du Paraguay recoltées par M. Balansa de 1874 à 1877. Mém. Soc. Sci. Nat. Cherbourg $21: 257-272$.

Bescherelle, E. (1885). Liste des mousses du Paraguay distribuées en 1884 par M. Balansa. Rev. Bryol. 2: 17-19.

Bescherelle, E. (1891a). Selectio Novorum Muscorum. J. Bot. (Morot) $5:$ 142-148.

Bescherelle, E. (1891b). Selectio Novorum Muscorum (suite). J. Bot. (Morot) 5: 252-255.

Bescherelle, E. (1891c). Selectio Novorum Muscorum (fin). J. Bot. (Morot) 5: 342-350.

Bowers, F. D. (1970). High elevation mosses of Costa Rica. J. Hattori Bot. Lab. 33: 7-35.

Brummitt, R. K. \& C. E. Powell (1992). Authors of Plant Names. Royal Botanic Gardens, Kew.

BucK, W. R. (1982). On Meiothecium (Sematophyllaceae). Contr. Univ. Michigan Herb. 15: 137-140.

Buck, W. R. (1983). A synopsis of the South American taxa of Fabronia. Brittonia 35: 248-254.

Buck, W. R. (1985). A preliminary list of the mosses of Paraguay. Candollea 40: 201-209.

BucK, W. R. (1987). Taxonomic and nomenclatural rearrangement in the Hookeriales with notes on West Indian taxa. Brittonia 39: 210-224.

Buck, W. R. \& R. R. IRELAND (1985). A reclassification of the Plagiotheciaceae. Nova Hedwigia 41: 89-125.

Buck, W. R. \& R. R. IRELAND (1989). Plagiotheciaceae. Fl. Neotrop. Monogr. 50: 1-20.

CÂmara, P. E. A. S. \& R. E. Magill (2009). A review of Dimerodontium (Fabroniaceae). Bryologist 112: 301-307.

CaO, T., S.-L. Guo \& Y.-M. Zhang (2001). Distribution of Ptychomitrium muelleri (Bryopsida), with its synonyms. Bryologist 104: 522-526.

Crosby, M. R. (1999). Vade mecum bryologiae I. Abbreviations for bryological nomenclatural literature, with full titles and other useful information. Contr. Hedw. Soc. 2: 1-108.

Crosby, M. R., R. E. Magill \& S. He (1999). A Checklist of the Mosses. Missouri Botanical Garden, St. Louis, U. S. A.

Crum, H. (1972). A Taxonomic account of the Erpodiaceae. Nova Hedwigia 23: 201-224.

CRUM, H. \& W. R. BUCK (1988). A contribution to the Sphagnum (Sphagnaceae) flora of Paraguay. Brittonia 40: 188-194. 
Crum, H. \& W. C. Steere (1957). The mosses of Porto Rico and the Virgin Islands. Sci. Surv. Porto Rico \& Virgin Islands 7: 395-599.

Dismier, G. (1910). Révision des Philonotis de l'Amérique. Mém. Soc. Bot. France 17: 1-37.

Fourier, B. (2005). Les découvertes de Balansa se sont étendues jusqu'aux antipodes. Bull. Assoc. Parcs Bot. France 40: 2-9.

FrAHM, J.-P. (1982). Grossdisjunktionen von Arealen sudamerikanischer und afrikanischer Campylopus-arten. Lindbergia 8: 45-52.

GrifFIn, D. I. (1994). Bartramiaceae. In: SHARP, A. J. \& al. (ed.), Moss Flora of Mexico. Mem. New York Bot. Gard. 69: 537-574.

Grout, A. J. (1945). A revision of the North American species of Stereophyllum and Pilosium, with descriptions of some South American species. Bryologist 48: 60-70.

HyvöNEN, J. (1989). A synopsis of genus Pogonatum (Polytrichaceae, Musci). Acta Bot. Fenn. 138: 1-87.

IRELAND, R. R. (1991). A preliminary study of the moss genus Isopterygium in Latin America. Caldasia 16: 265-276.

Ireland, R. R. \& W. R. BucK (1994). Stereophyllaceae. Fl. Neotrop. Monogr. 65: 1-50.

Kruijer, J. D. (2002). Hypopterygiaceae of the World. Blumea, Suppl. 13: $1-388$

Lawton, E. (1953). Lorentziella, a moss genus new to North America. Bull. Torrey Bot. Club 80: 279-288.

MaJestyK, P. (2009). Revision of Erythrodontium (Endodontaceae). Bryologist 112: 804-822.

Manuel, M. G. (1977). Studies in Cryphaeaceae IV. New combinations in Schoenobryum. Bryologist 80: 522-524.

MüLLER, C. (1887). Erpodiaceae quatuor novae. Flora 70: 446-450.

Müller, C. (1888). Musci Cleistocarpici novi. Flora 71: 1-13.

Müller, C. (1897). Prodromus Bryologiae Argentinicae atque regionum vicinarum. III. Hedwigia 36: 84-144.
MÜller, C. \& F. StePHANI (1887). Musci exotici. Rev. Bryol. 14: 56-58.

O'Shea, B. J. \& M. J. Price (2008). An updated checklist of the mosses of Paraguay. Trop. Bryol. 29: 6-40.

OchI, H. (1980). A revision of the neotropical Bryoideae, Musci (First part). J. Fac. Ed., Tottori Univ., Nat. Sci. 29: 49-154.

OchyrA, R. (1997). Leptotrichella replaces Microdus (Musci, Dicranaceae). Fragm. Flor. Geobot. 42: 559-565.

Pursell, R. A. (1994). Taxonomic notes on neotropical Fissidens. Bryologist 97: 253-271.

Pursell, R. A. (2007). Fissidentaceae. Fl. Neotrop. Monogr. 101: 1-279.

ReEse, W. D. (1978). The genus Syrrhopodon in the Americas II. The Limbate species. Bryologist 81: 189-225.

REIMERS, H. (1937). Die europaischen Haplocladium-Arten mit besonderer Berucksichtigung ihrere aussereuropaischen Verbreitung und ihrere Verwandtschaft. Hedwigia 76: 191-298.

Rushing, A. E. (1986). A revision of the genus Bruchia Schwaegr. (Musci). J. Hattori Bot. Lab. 60: 35-83.

STARK, L. R. (1987). A taxonomic monograph of Forsstroemia Lindb. (Bryopsida: Leptodontaceae). J. Hattori Bot. Lab. 63: 133-218.

Stone, I. G. (1997). A revision of Erpodiaceae with special reference to Australian taxa. J. Bryol. 19: 485-502.

VAn der WiJK, R., W. D. Margadant \& P. A. Florschütz (1959). Index Muscorum. Volume 1 (A-C). Regnum Veg. 17.

VAN DER WiJk, R., W. D. Margadant \& P. A. Florschütz (1964). Index Muscorum Volume 3 (Hypnum - H-O). Regnum Veg. 33.

VITT, D. H. (1980). The Genus Macrocoma I. Typification of names and taxonomy of the species. Bryologist 83: 405-436.

ZARDINI, E. (1993). Paraguay's floristic inventory. Res. \& Explor. (Washington, DC) 9: 128-131. 


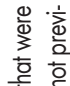

总产

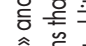

을

妾尊

E

(0)

응

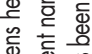

旁

边员. 응

응 흘

응

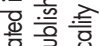

을 흥응

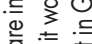

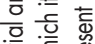

弯

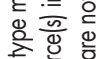

홍ㅎㅁ훙

흥 홓

훟 홓

㟧产 क

妾它递

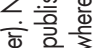

है o

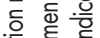

응 응

응 응

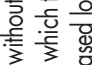

웡ㅎㅇ

응 등 음

을 흠

خे

인

홀

튼 드ㄴㅡㅡ 등

휴

ग

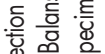

흥 후

응

든

웡ㅎㄴ항

产全放

ब잉

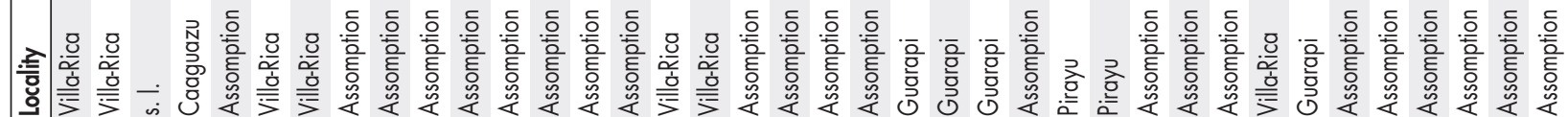

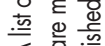

임응

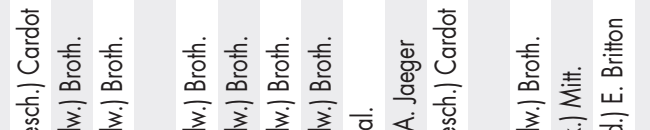

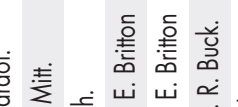

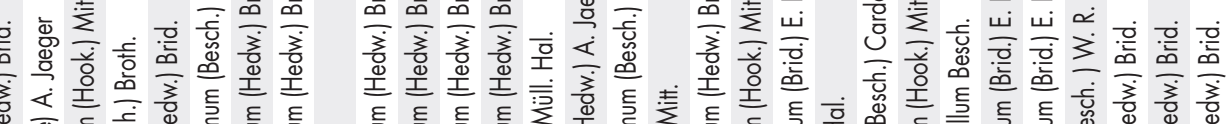

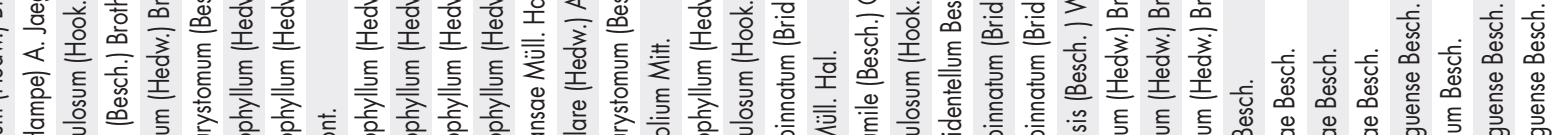
$\cong$ E

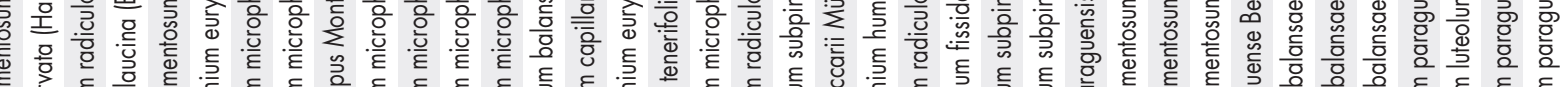

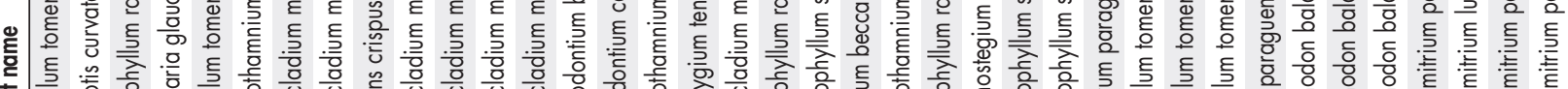

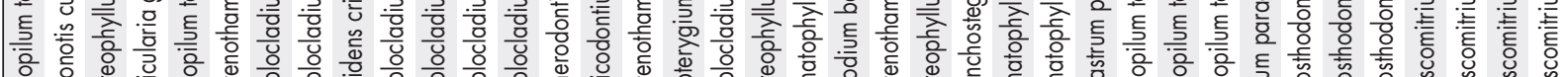

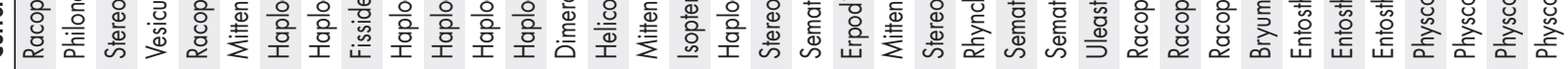

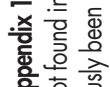

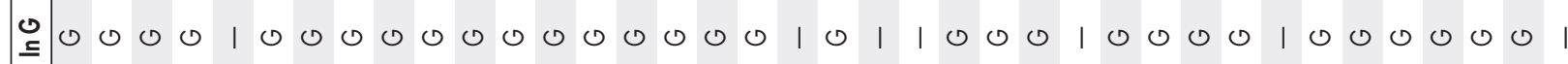

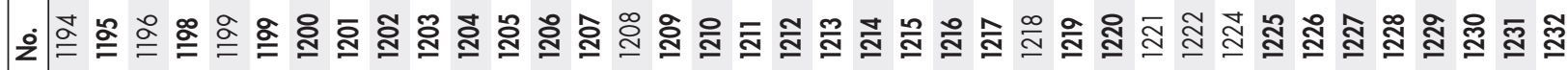




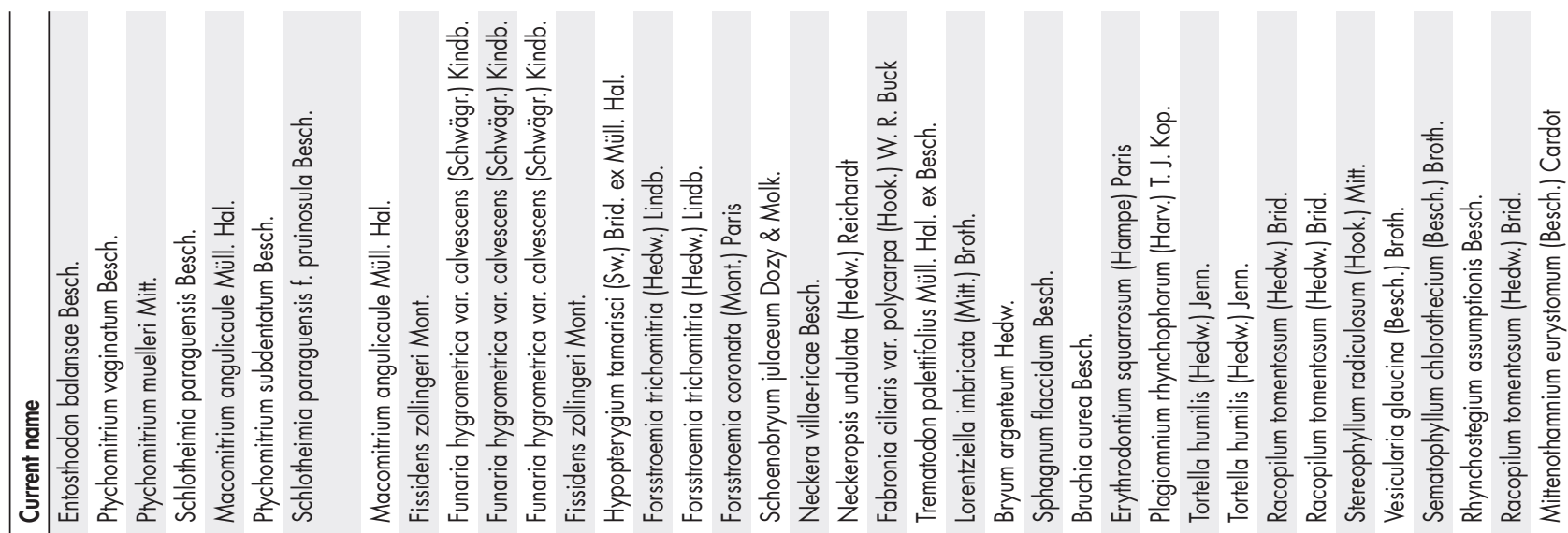

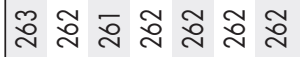

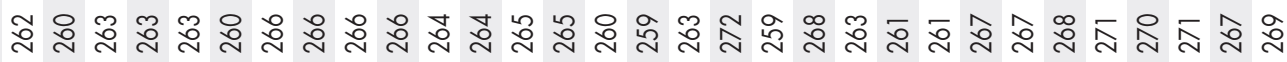

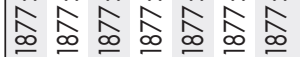

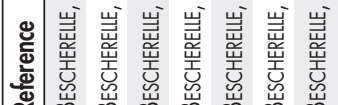

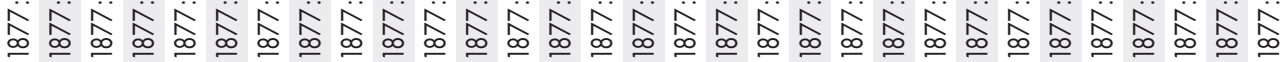

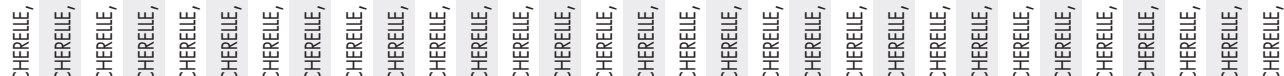

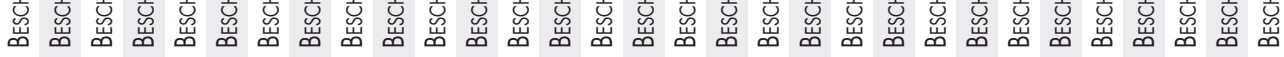
总总总总总总总

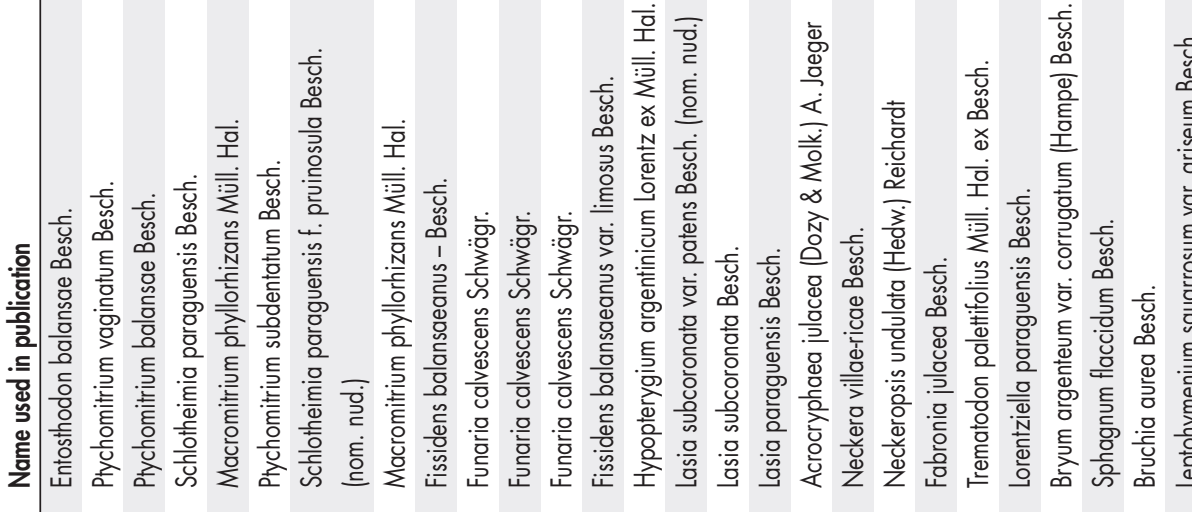

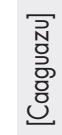

券

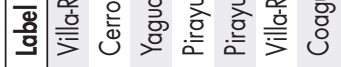
\begin{tabular}{l|lllllll}
0 & 1 & 0 & 0 & 0 & 0 & 1 & 1
\end{tabular}

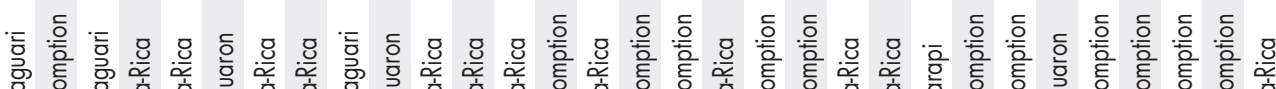

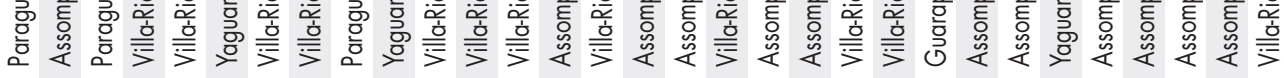
$\begin{array}{llllllllllllllllllllllllllllll}1 & 0 & 1 & 1 & 1 & 0 & 1 & 1 & 1 & 0 & 1 & 1 & 0 & 0 & 0 & 0 & 0 & 0 & 0 & 1 & 1 & 1 & 1 & 1 & 1 & 1 & 1 & 1 & 1 & 1\end{array}$ 


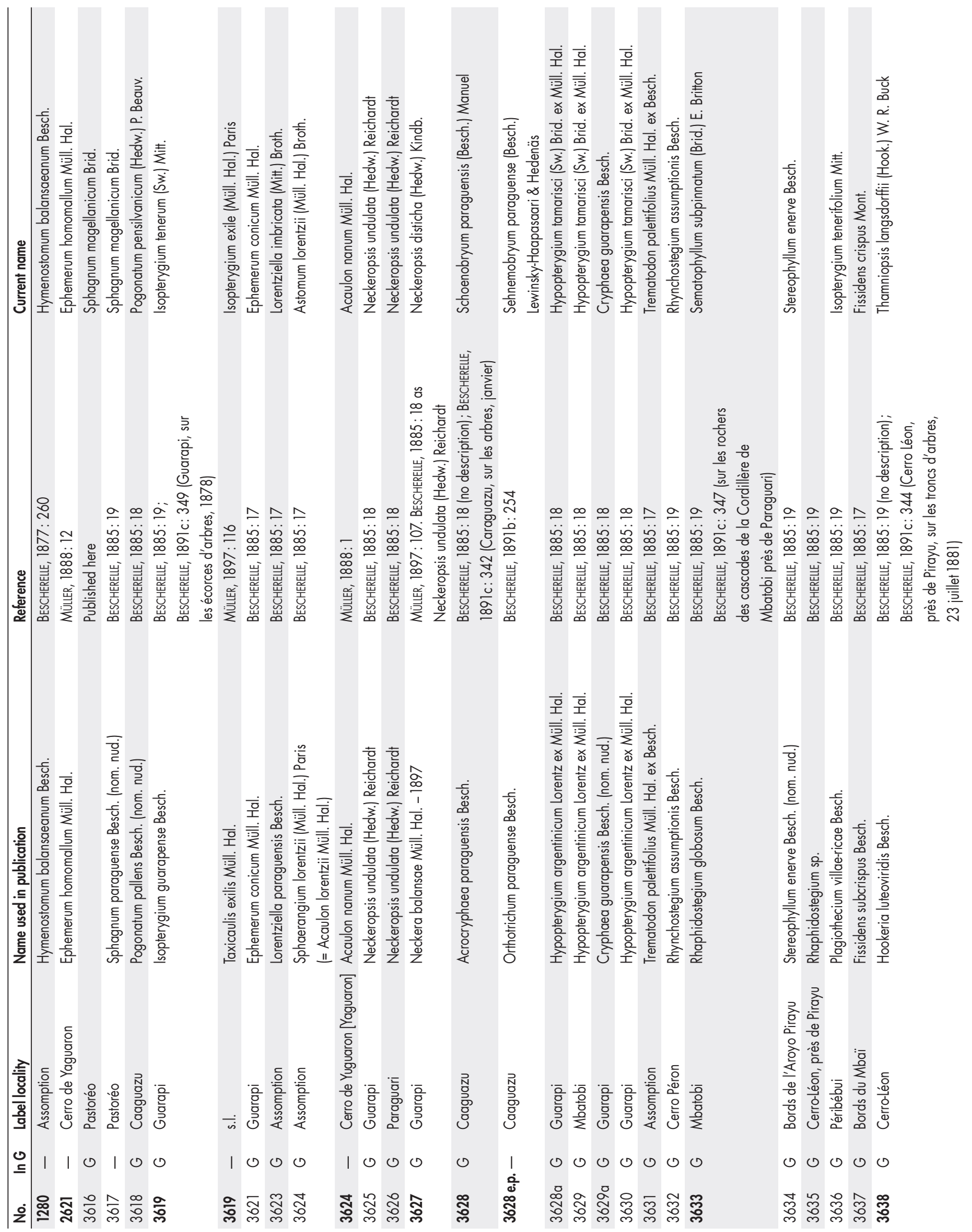



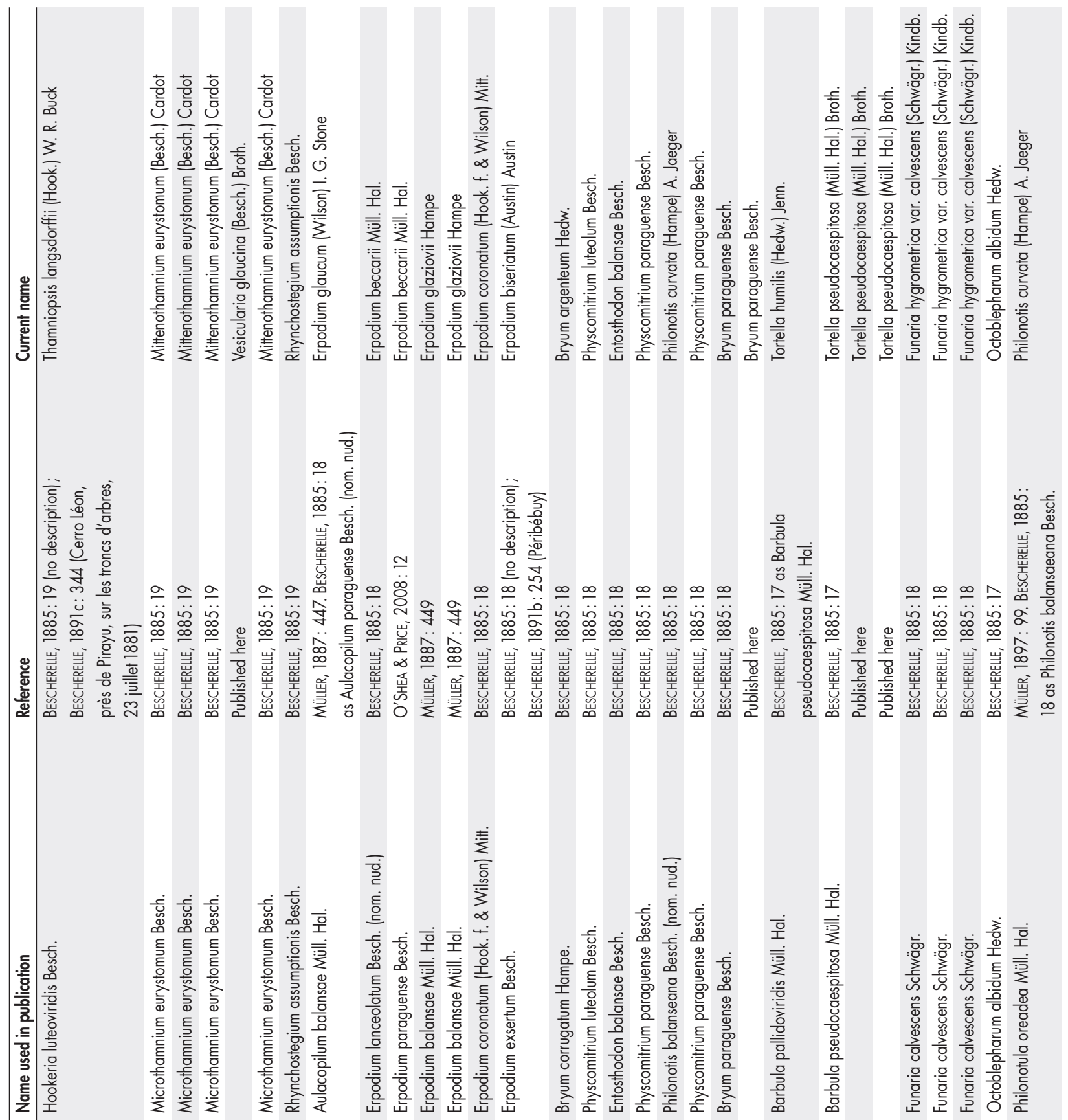

$\frac{\sqrt{2}}{\frac{9}{3}}$

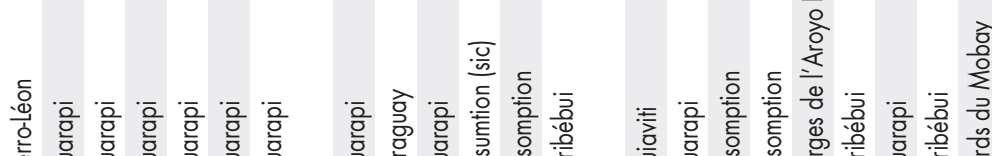

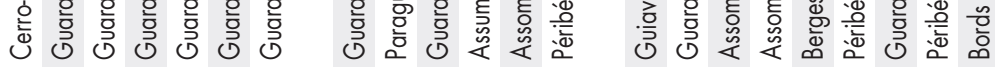
$\begin{array}{llllllll}0 & 1 & 0 & 0 & 0 & 0 & 0\end{array}$

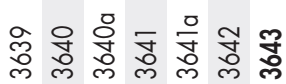

$\begin{array}{llllllll}0 & 1 & 0 & 0 & 1 & 0\end{array}$

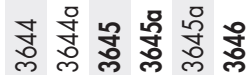

$\begin{array}{llllllllll}0 & 0 & 0 & 0 & 1 & 0 & 0 & 0 & 0\end{array}$

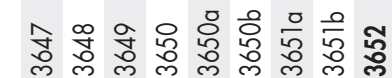

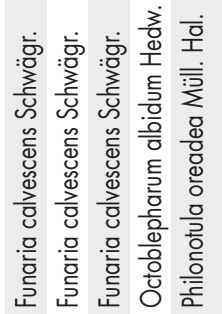

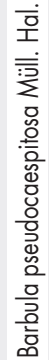<smiles>[13CH]=[131In]</smiles>

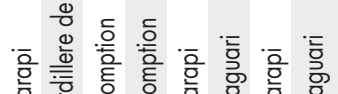

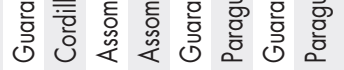
00000000

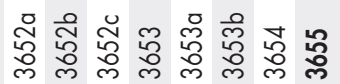




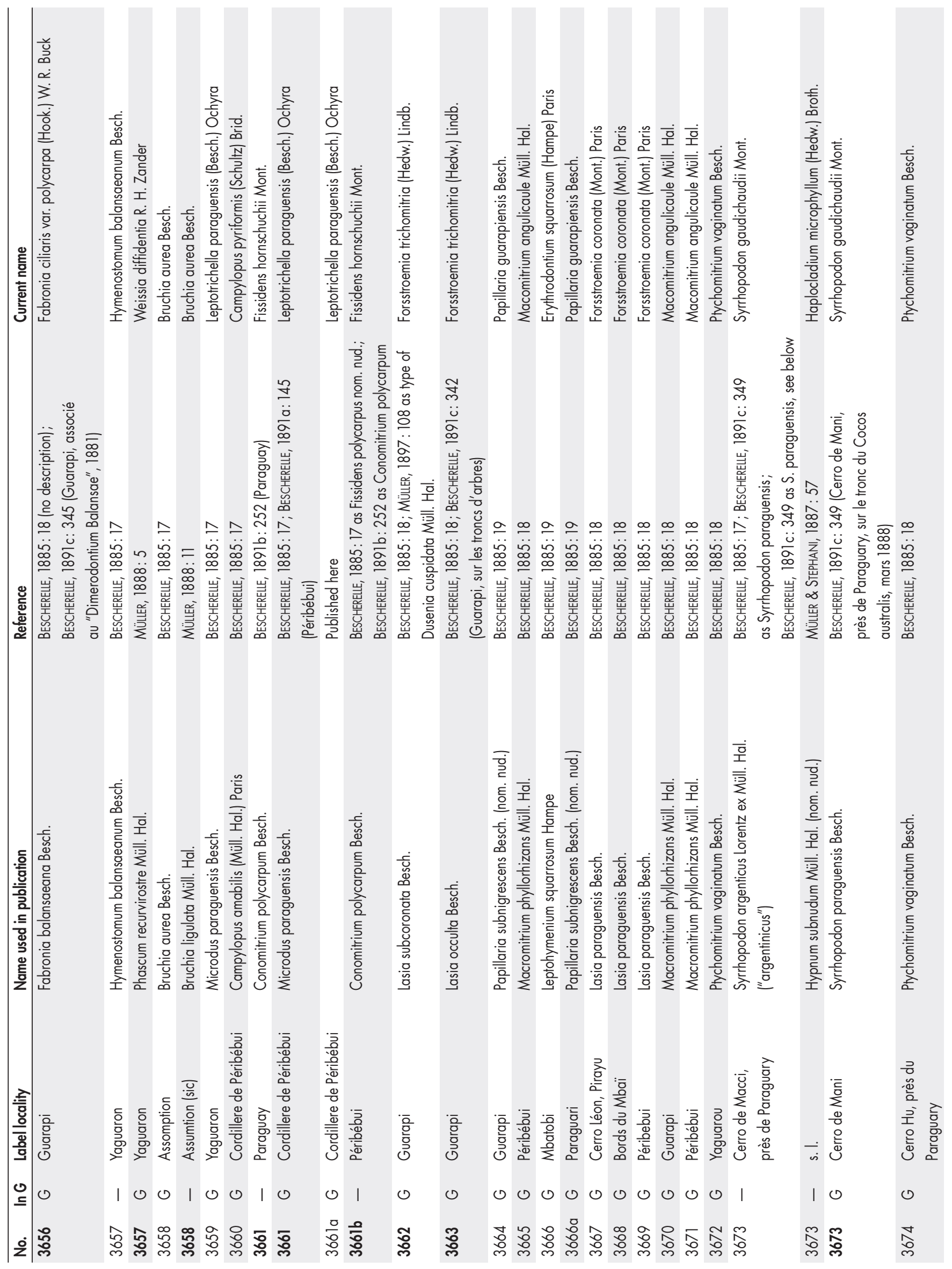



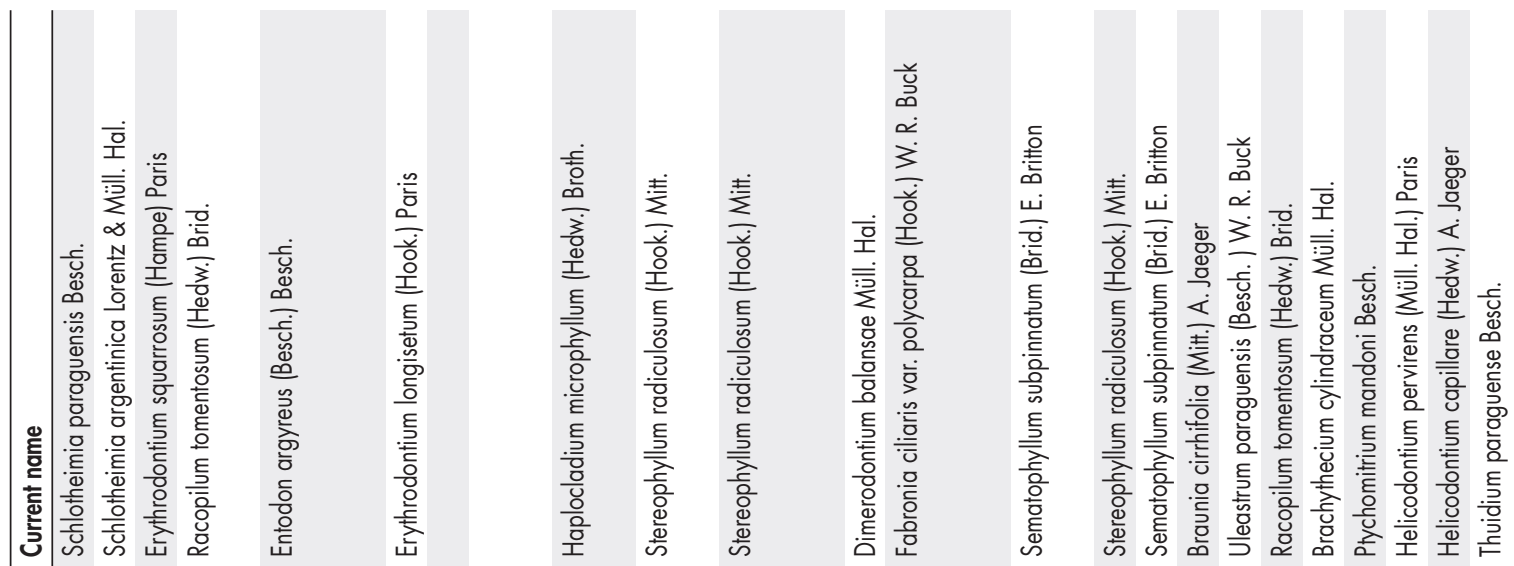

กิ

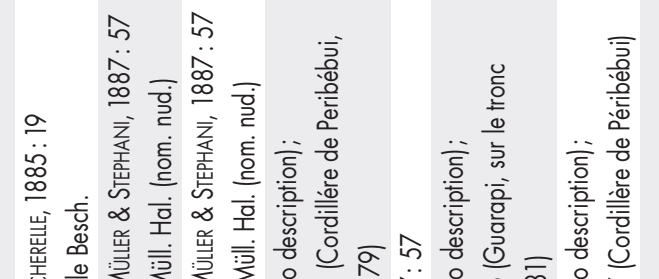

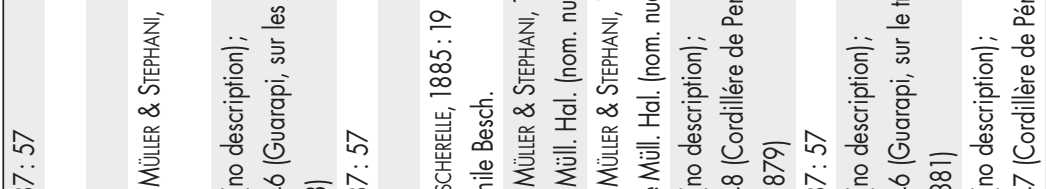

$\stackrel{\infty}{\infty} \propto \stackrel{\sum_{\infty}^{\infty}}{\infty}$

을

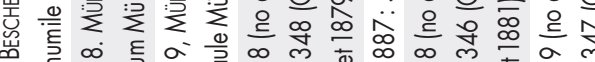

要

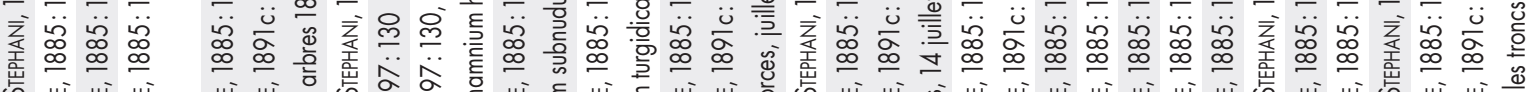

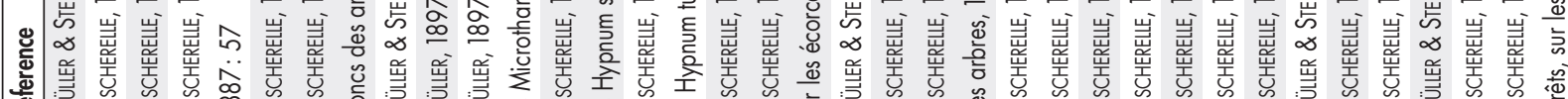

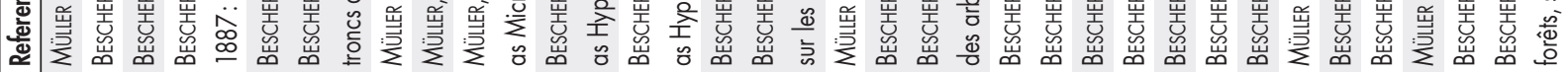

홀

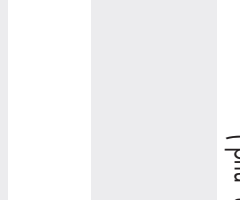

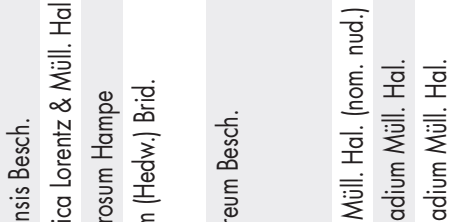

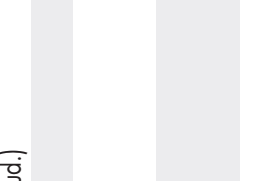




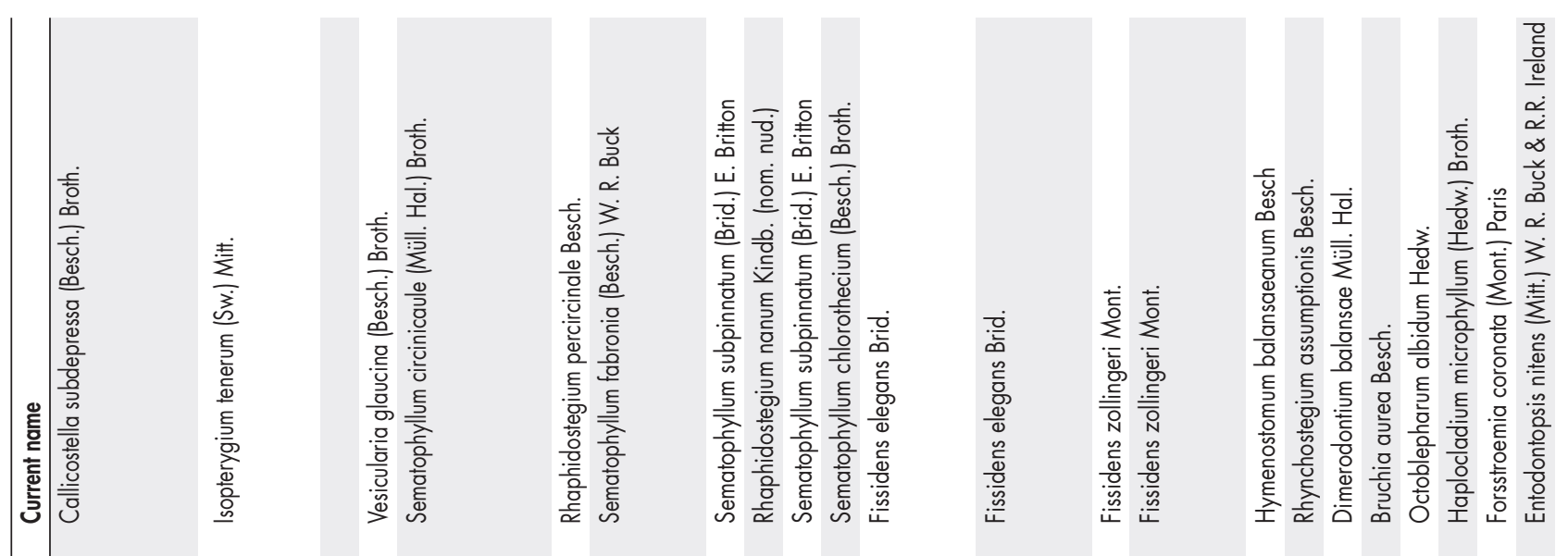

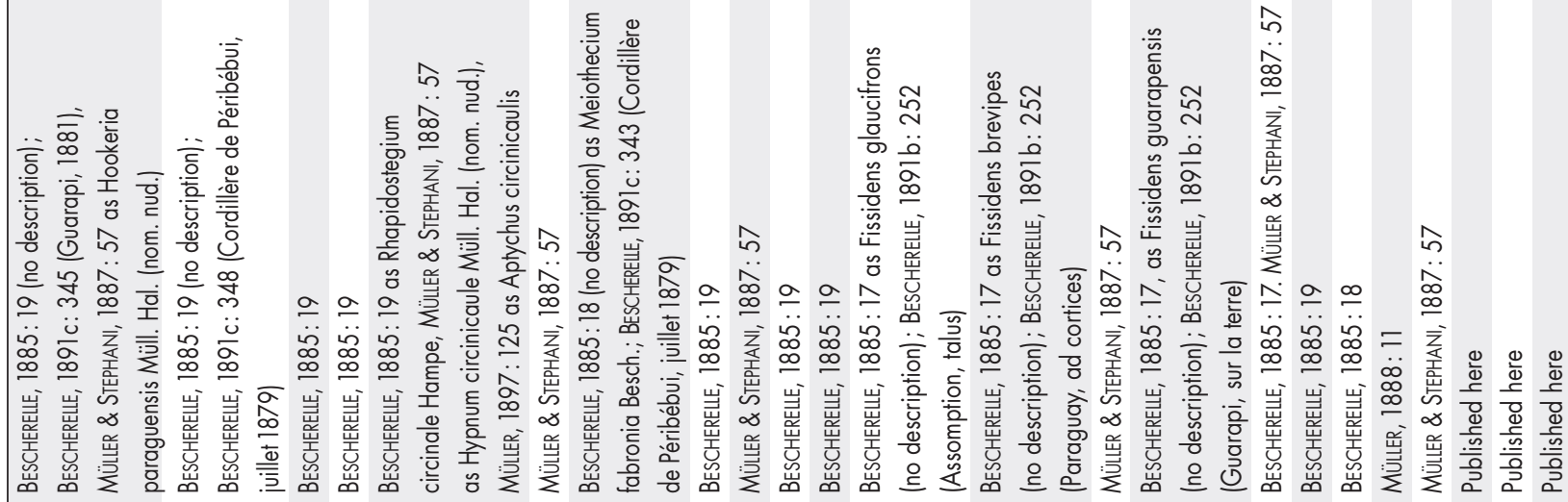
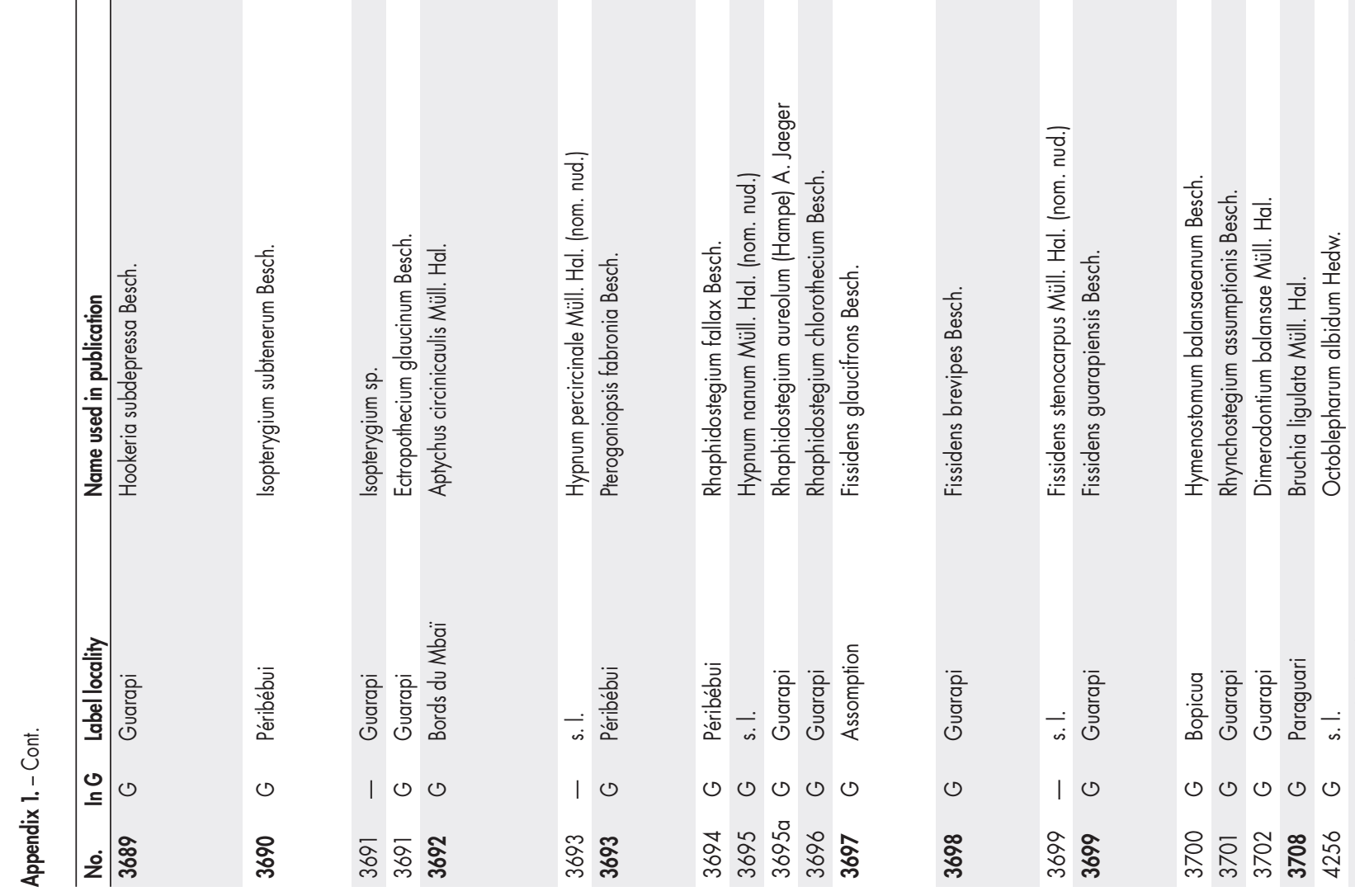

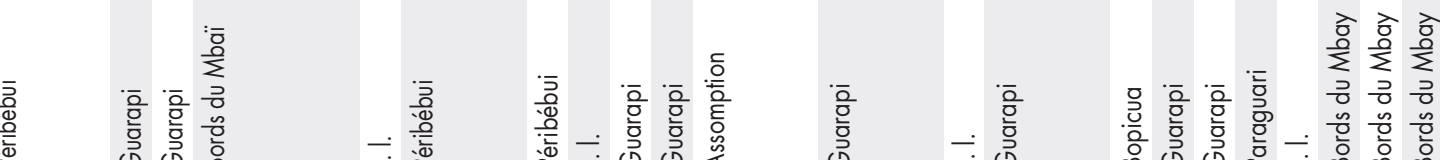

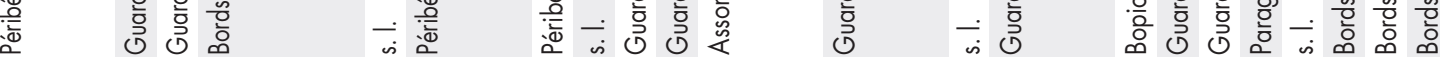

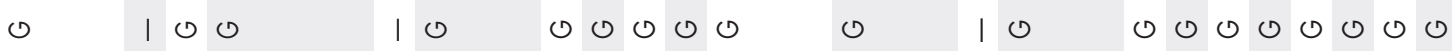

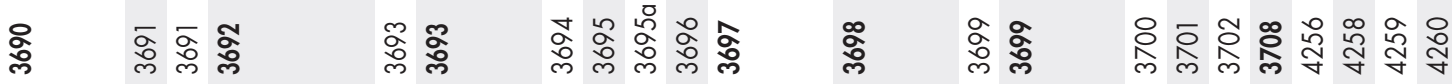




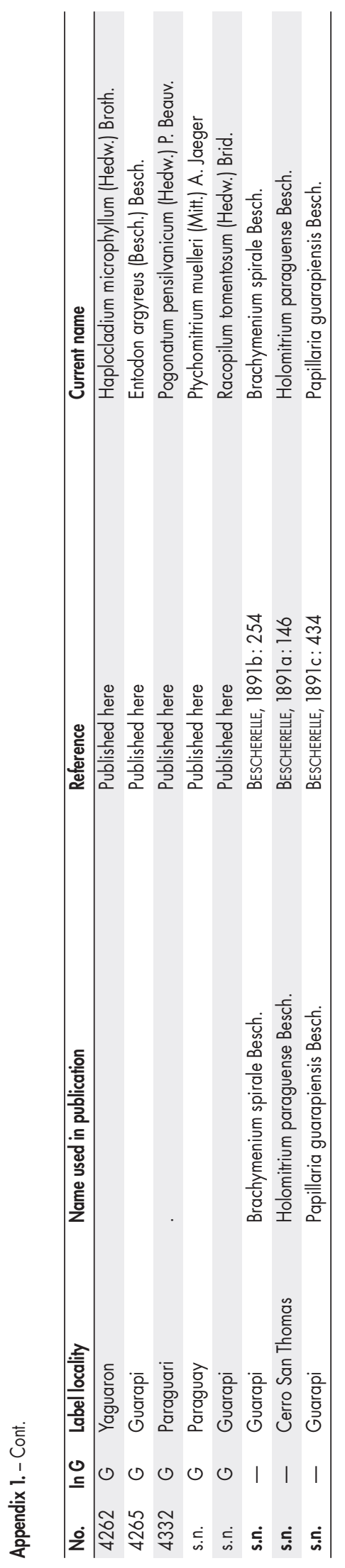

